Supporting Information for:

\title{
A Gold(I)-Catalyzed Propargyl Claisen Rearrangement
}

Benjamin D. Sherry and F. Dean Toste

\section{Center for New Directions in Organic Synthesis, Department of Chemistry, University of California, Berkeley, California 94720}

General Information: Unless otherwise noted all commercial materials were used without further purification. Claisen rearrangements were conducted in two dram vials equipped with a magnetic stir bar and fitted with a threaded cap. Dichloromethane $\left(\mathrm{CH}_{2} \mathrm{Cl}_{2}\right)$ and methanol $(\mathrm{MeOH})$ were obtained from EMD. Silver tetrafluoroborate $\left(\mathrm{AgBF}_{4}\right)$, silver trifluoromethanesulfonate $(\mathrm{AgOTf})$, and sodium borohydride $\left(\mathrm{NaBH}_{4}\right)$ were obtained from Aldrich Chemical Company. Triphenylphosphinegold(I) chloride $\left(\mathrm{Ph}_{3} \mathrm{PAuCl}\right)$ was prepared according to the method of Bruce. ${ }^{1}$ Tris(triphenylphosphinegold)-oxonium tetrafluoroborate $\left.\left(\left[\left(\mathrm{Ph}_{3} \mathrm{PAu}\right)_{3} \mathrm{O}\right)\right] \mathrm{BF}_{4}\right)$ was prepared according to the method of Sharp. ${ }^{2}$ Bis(triphenylphosphine)gold(I) tetrafluoroborate $\left(\left[\left(\mathrm{Ph}_{3} \mathrm{P}\right)_{2} \mathrm{Au}\right] \mathrm{BF}_{4}\right)$ was prepared according to the method of Westland. ${ }^{3}$-Aurated acetaldehyde $\left(\mathrm{Ph}_{3} \mathrm{PAuCH}_{2} \mathrm{CHO}\right)$ was prepared according to the method of Nesmeyanov. ${ }^{4}$ Vinyl ethers $\mathbf{1},(\mathbf{R})-\mathbf{4 a}, \mathbf{( R )}-\mathbf{4} \mathbf{j}$, and (R)-4n were prepared by reduction of the corresponding ynone according to the method of Midland, ${ }^{5}$ followed by vinylation of the resulting propargyl alcohol according to the method of Yamamoto. ${ }^{6}$ Vinyl ethers 4a-n were prepared from the corresponding propargyl alcohol according to the method of Yamamoto. ${ }^{6}$ Thin-layer chromatography (TLC) analysis of reaction mixtures was performed using Merck silica gel $60 \mathrm{~F}_{254}$ TLC plates and visualized by UV and permanganate staining. Flash column chromatography was carried out on Merck 60 silica gel (32-63_m). ${ }^{1} \mathrm{H}$ and ${ }^{13} \mathrm{C}$ NMR spectra were recorded with Bruker DRX-500, AVB-400, AVQ-400, and AV-300 spectrometers and referenced to $\mathrm{CDCl}_{3}$ unless otherwise noted. Enantiomeric excess was determined either on an Agilent 1100 Series Chiral HPLC with detection at 210, 254, and $280 \mathrm{~nm}$ or a Shimadzu VP Series Chiral HPLC with detection at 210, 254, and $280 \mathrm{~nm}$. Mass spectral and analytical data were obtained via the Micro-Mass/Analytical Facility operated by the College of Chemistry, University of California, Berkeley.

Catalyst Optimization Experiments:

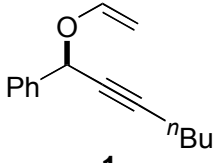

1

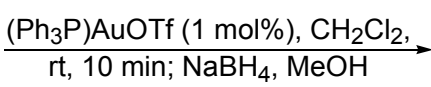

rt, $10 \mathrm{~min} ; \mathrm{NaBH}_{4}, \mathrm{MeOH}$

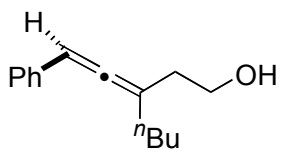

2

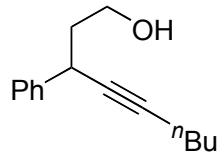

To a solution of $1(0.100 \mathrm{~g}, 0.467 \mathrm{mmol}, 95 \%$ ee $)$ in $\mathrm{CH}_{2} \mathrm{Cl}_{2}(1.3 \mathrm{~mL})$ was added $\mathrm{Ph}_{3} \mathrm{PAuCl}(2.3 \mathrm{mg}, 4.7 \square \mathrm{mol})$ followed by AgOTf (1.2 mg, $4.7 \square \mathrm{mol})$ and the reaction mixture was left to stir at $\mathrm{rt}$ for $10 \mathrm{~min}$. The solution was diluted with $\mathrm{MeOH}(2.6 \mathrm{~mL}), \mathrm{NaBH}_{4}(17.7 \mathrm{mg}, 0.467 \mathrm{mmol})$ was added, and the reaction mixture was maintained at $\mathrm{rt}$ for $30 \mathrm{~min}$. The red solution was concentrated and a crude ${ }^{1} \mathrm{H}$ NMR $\left(400 \mathrm{MHz}, \mathrm{C}_{6} \mathrm{D}_{6}\right)$ spectrum was obtained: A 1.7:1.0 ratio of $\mathbf{2 : 3}$ was observed. The crude mixture was purified by flash column chromatography (9:1 hexanes:EtOAc eluent) to afford homoallenic alcohol $2\left(40 \mathrm{mg}, 40 \%\right.$ yield, $\mathrm{R}_{f}=0.20$ in 9:1 hexanes:EtOAc) as a colorless oil and alcohol 3 (24 mg, 24\% yield, $R_{f}=0.20$ in 9:1 hexanes:EtOAc) as a colorless oil. Characterization for 2: Spectral data were consistent with those reported below; Enantiomeric purity was determined by chiral HPLC analysis, Chiralcel OD column, 97:3 hexanes:isopropanol, $1 \mathrm{~mL} / \mathrm{min}$; $\mathrm{t}_{\mathrm{R}} 9.30 \mathrm{~min}$ (minor), $12.76 \mathrm{~min}$ (major): $83 \%$ ee. Characterization for 3: ${ }^{1} \mathrm{H}$ NMR $\left(400 \mathrm{MHz}, \mathrm{CDCl}_{3}\right) \square 7.37(\mathrm{~d}, 2 \mathrm{H}, J=7.2 \mathrm{~Hz}), 7.33-7.26(\mathrm{~m}, 3 \mathrm{H}), 3.89-3.80(\mathrm{~m}$, $2 \mathrm{H}), 3.77-3.71(\mathrm{~m}, 2 \mathrm{H}), 2.23(\mathrm{td}, 2 \mathrm{H}, J=6.8,2.0 \mathrm{~Hz}), 2.02-1.87(\mathrm{~m}, 2 \mathrm{H}), 1.80($ br s, $1 \mathrm{H}), 1.55-1.48(\mathrm{~m}, 2 \mathrm{H}), 1.45-$ 1.31 (m, 2H), 0.91 (t, $2 \mathrm{H}, J=7.2 \mathrm{~Hz}) ;{ }^{13} \mathrm{C}$ NMR (100 MHz, $\left.\mathrm{CDCl}_{3}\right) \square 142.3,128.5,127.4,126.7,84.1,81.1,61.1$, 41.3, 34.7, 31.1, 22.0, 18.5, 13.7; HRMS $\left(\mathrm{EI}^{+}\right)$calc'd for $\left[\mathrm{C}_{15} \mathrm{H}_{20} \mathrm{O}\right]^{+}: \mathrm{m} / z 216.1514$, found 216.1517 .
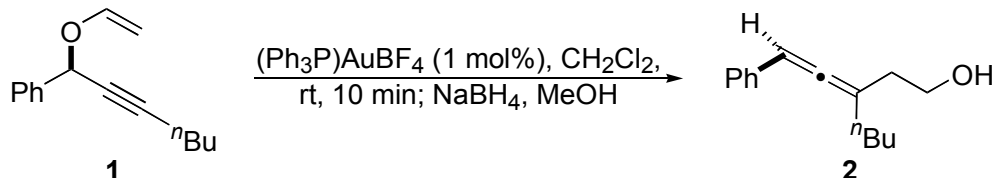

To a suspension of $\mathrm{AgBF}_{4}(0.9 \mathrm{mg}, 4.7 \square \mathrm{mol})$ in $\mathrm{CH}_{2} \mathrm{Cl}_{2}(0.4 \mathrm{~mL})$ was added $\mathrm{Ph}_{3} \mathrm{PAuCl}\left(2.3 \mathrm{mg}, 4.7 \_\mathrm{mol}\right)$ and the resulting cloudy mixture as stirred rapidly at $\mathrm{rt}$ for $5 \mathrm{~min}$. The mixture was filtered through a cotton plug into a 
stirred solution of $1(0.100 \mathrm{~g}, 0.467 \mathrm{mmol}, 95 \%$ ee $)$ in $\mathrm{CH}_{2} \mathrm{Cl}_{2}(0.9 \mathrm{~mL})$ and the resulting mixture was left to stir at $\mathrm{rt}$ for $10 \mathrm{~min}$. The reaction mixture was diluted with $\mathrm{MeOH}(2.6 \mathrm{~mL}), \mathrm{NaBH}_{4}(17.7 \mathrm{mg}, 0.467 \mathrm{mmol})$ was added, and the solution was left to stir at $\mathrm{rt}$ for $1 \mathrm{~h}$. The red solution was concentrated and the crude oil was purified by flash column chromatography (9:1 hexanes:EtOAc eluent) to afford the desired homoallenic alcohol 2 (86 $\mathrm{mg}, 85 \%$ yield, $\mathrm{R}_{f}=0.38$ in 3:1 hexanes:EtOAc) as a colorless oil: ${ }^{1} \mathrm{H}$ NMR $\left(400 \mathrm{MHz}, \mathrm{CDCl}_{3}\right)$ 7 7.35-7.31 (m, 4H), 7.25-7.19 (m, $1 \mathrm{H}), 6.23$ (quintet, $1 \mathrm{H}, \mathrm{J}=3.2 \mathrm{~Hz}$ ), $3.80(\mathrm{t}, 2 \mathrm{H}, J=6.4 \mathrm{~Hz}), 2.47-2.32(\mathrm{~m}, 2 \mathrm{H}), 2.19-2.13(\mathrm{~m}, 2 \mathrm{H}), 1.79(\mathrm{br} \mathrm{s}, 1 \mathrm{H})$, 1.57-1.48 (m, 2H), 1.38 (sextet, $2 \mathrm{H}, J=7.2 \mathrm{~Hz}), 0.93(\mathrm{t}, 3 \mathrm{H}, J=7.2 \mathrm{~Hz}) ;{ }^{13} \mathrm{C}$ NMR $\left(100 \mathrm{MHz}, \mathrm{CDCl}_{3}\right) \square 201.9$, 135.4, 128.7, 126.8, 126.5, 105.8, 96.1, 60.9, 35.9, 32.8, 29.8, 22.5, 13.9; HRMS (EI ${ }^{+}$) calc'd for $\left[\mathrm{C}_{15} \mathrm{H}_{20} \mathrm{O}\right]^{+}: \mathrm{m} / z$ 216.1514, found 216.1513; Enantiomeric purity was determined by chiral HPLC analysis, Chiralcel OD column, 97:3 hexanes:isopropanol, $1 \mathrm{~mL} / \mathrm{min} ; \mathrm{t}_{\mathrm{R}} 14.53 \mathrm{~min}$ (minor), $27.10 \mathrm{~min}$ (major): $4 \%$ ee.
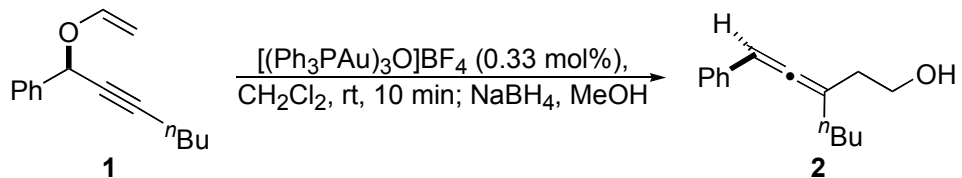

To a solution of $1(0.100 \mathrm{~g}, 0.467 \mathrm{mmol}, 95 \%$ ee $)$ in $\mathrm{CH}_{2} \mathrm{Cl}_{2}(1.3 \mathrm{~mL})$ was added $\left.\left[\left(\mathrm{Ph}_{3} \mathrm{PAu}\right)_{3} \mathrm{O}\right)\right] \mathrm{BF}_{4}(2.3 \mathrm{mg}, 1.6$ _mol) and the resulting solution was left to stir at $\mathrm{rt}$ for $10 \mathrm{~min}$. The reaction mixture was diluted with $\mathrm{MeOH}(2.6$ $\mathrm{mL}), \mathrm{NaBH}_{4}(17.7 \mathrm{mg}, 0.467 \mathrm{mmol})$ was added, and the solution was left to stir at $\mathrm{rt}$ for $1 \mathrm{~h}$. The red solution was concentrated and the crude oil was purified by flash column chromatography ( $9: 1$ hexanes:EtOAc eluent) to afford the desired homoallenic alcohol 2 (92 mg, 91\% yield, $\mathrm{R}_{f}=0.14$ in 9:1 hexanes:EtOAc) as a colorless oil: Spectral data were consistent with those reported for 2; Enantiomeric purity was determined by chiral HPLC analysis, Chiralcel OD column, $97: 3$ hexanes:isopropanol, $1 \mathrm{~mL} / \mathrm{min}$; $\mathrm{t}_{\mathrm{R}} 14.33 \mathrm{~min}$ (minor), $26.92 \mathrm{~min}$ (major): 90\% ee.

General Procedure for the Au(I)-Catalyzed Claisen Rearrangement: To a solution of propargyl vinyl ether (1 equiv) in $\mathrm{CH}_{2} \mathrm{Cl}_{2}(0.35 \mathrm{M})$ was added $\left.\left[\left(\mathrm{Ph}_{3} \mathrm{PAu}\right)_{3} \mathrm{O}\right)\right] \mathrm{BF}_{4}(1 \mathrm{~mol} \%)$ and the resulting mixture was maintained at $\mathrm{rt}$ until TLC analysis indicated consumption of the starting material. The reaction mixture was diluted with $\mathrm{MeOH}(2 \mathrm{x}$ the volume of $\mathrm{CH}_{2} \mathrm{Cl}_{2}$ ) and $\mathrm{NaBH}_{4}$ (1.0 equiv) was added. The resulting red solution was maintained at $\mathrm{rt}$ for a minimum of $1 \mathrm{~h}$, concentrated, and purified by flash column chromatography. For reactions carried out on a scale greater than $0.500 \mathrm{~g}$ the reaction mixture after reduction was diluted with $\mathrm{H}_{2} \mathrm{O}$, extracted with $\mathrm{Et}_{2} \mathrm{O}(3 \mathrm{x})$, washed with brine (1x), dried over $\mathrm{MgSO}_{4}$, filtered, and concentrated. The crude residue was purified by flash column chromatography.<smiles>OCC/C=C/c1ccccc1</smiles>

5a (Table 1, entry 1$)$

The crude mixture was purified by flash column chromatography (7:1 hexanes:EtOAc eluent) to afford the desired homoallenic alcohol (45 mg, 90\% yield, $\mathrm{R}_{f}=0.24$ in 3:1 hexanes:EtOAc) as a colorless oil: ${ }^{1} \mathrm{H}$ NMR (400 MHz, $\left.\mathrm{CDCl}_{3}\right) \square 7.33-7.29(\mathrm{~m}, 4 \mathrm{H}), 7.23-7.17(\mathrm{~m}, 1 \mathrm{H}), 6.19(\mathrm{~m}, 1 \mathrm{H}), 5.60(\mathrm{q}, 1 \mathrm{H}, J=6.8 \mathrm{~Hz}), 3.77(\mathrm{t}, 2 \mathrm{H}, J=6.4 \mathrm{~Hz})$, 2.39 (m, 2H), 1.78 (br s, 1H); ${ }^{13} \mathrm{C}$ NMR (100 MHz, $\left.\mathrm{CDCl}_{3}\right) \square$ 205.7, 134.4, 128.6, 126.9, 126.6, 95.1, 91.5, 61.9, 32.0 .

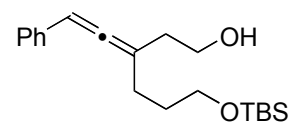

5b (Table 1, entry 2)

The crude mixture was purified by flash column chromatography ( $9: 1$ hexanes:EtOAc eluent) to afford the desired homoallenic alcohol ( $89 \mathrm{mg}, 89 \%$ yield, $\mathrm{R}_{f}=0.12$ in 9:1 hexanes:EtOAc) as a colorless oil: ${ }^{1} \mathrm{H}$ NMR (400 MHz, $\left.\mathrm{CDCl}_{3}\right) \square 7.28-7.22(\mathrm{~m}, 4 \mathrm{H}), 7.17-7.13(\mathrm{~m}, 1 \mathrm{H}), 6.18$ (quintet, $\left.1 \mathrm{H}, J=3.2 \mathrm{~Hz}\right), 3.74(\mathrm{t}, 2 \mathrm{H}, J=6.4 \mathrm{~Hz}), 3.61(\mathrm{t}, 2 \mathrm{H}$, $J=6.4 \mathrm{~Hz}), 2.39-2.28(\mathrm{~m}, 2 \mathrm{H}), 2.18-2.12(\mathrm{~m}, 2 \mathrm{H}), 1.84(\mathrm{br} \mathrm{s}, 1 \mathrm{H}), 1.72-1.65(\mathrm{~m}, 2 \mathrm{H}), 0.86(\mathrm{~s}, 9 \mathrm{H}), 0.00(\mathrm{~s}, 6 \mathrm{H}) ;{ }^{13} \mathrm{C}$ NMR $\left(100 \mathrm{MHz}, \mathrm{CDCl}_{3}\right) \square 201.7,135.2,128.6,126.8,126.4,105.5,96.4,62.6,60.8,36.1,30.6,29.1,25.9,18.3$, 5.3; HRMS ( $\left.\mathrm{EI}^{+}\right)$calc'd for $\left[\mathrm{C}_{20} \mathrm{H}_{32} \mathrm{O}_{2} \mathrm{Si}^{+}: \mathrm{m} / z\right.$ 332.2171, found 332.2174; Anal calc'd for $\mathrm{C}_{20} \mathrm{H}_{32} \mathrm{O}_{2} \mathrm{Si}: \mathrm{C}, 72.23$; $\mathrm{H}$, 9.70; found: C, 71.76; H, 9.91. 


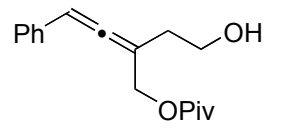

5c (Table 1 , entry 3$)$

The crude mixture was purified by flash column chromatography (4:1 hexanes:EtOAc eluent) to afford the desired homoallenic alcohol $\left(82 \mathrm{mg}, 81 \%\right.$ yield, $\mathrm{R}_{f}=0.21$ in $3: 1$ hexanes:EtOAc) as a colorless oil: ${ }^{1} \mathrm{H} \mathrm{NMR}(400 \mathrm{MHz}$, $\left.\mathrm{CDCl}_{3}\right) \square 7.30-7.26(\mathrm{~m}, 4 \mathrm{H}), 7.23-7.18(\mathrm{~m}, 1 \mathrm{H}), 6.32$ (quintet, $\left.1 \mathrm{H}, J=2.4 \mathrm{~Hz}\right), 4.69(\mathrm{dd}, 1 \mathrm{H}, J=16.0,2.8 \mathrm{~Hz}), 4.63$ $(\mathrm{dd}, 1 \mathrm{H}, J=14.4,2.8 \mathrm{~Hz}), 3.81(\mathrm{t}, 3 \mathrm{H}, J=6.0 \mathrm{~Hz}), 2.39-2.37(\mathrm{~m}, 2 \mathrm{H}), 2.10$ (br s, 1H), $1.15(\mathrm{~s}, 9 \mathrm{H}) ;{ }^{13} \mathrm{C} \mathrm{NMR}(100$ $\left.\mathrm{MHz}, \mathrm{CDCl}_{3}\right) \square 202.4,178.3,133.9,128.7,127.3,126.8,102.2,97.8,63.8,60.9,38.8,33.0,27.1$; $\left.\mathrm{HRMS}_{(\mathrm{EI}}^{+}\right)$ calc'd for $\left[\mathrm{C}_{17} \mathrm{H}_{22} \mathrm{O}_{3}\right]^{+}: \mathrm{m} / z$ 274.1569, found 274.1562; Anal calc'd for $\mathrm{C}_{17} \mathrm{H}_{22} \mathrm{O}_{3}$ : C, 74.42; H, 8.08; found: C, 74.12; $\mathrm{H}, 8.11$.

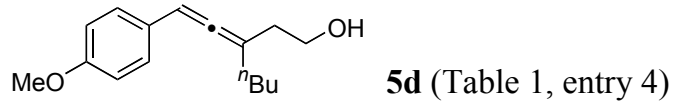

The crude mixture was purified by flash column chromatography (9:1 hexanes:EtOAc eluent) to afford the desired homoallenic alcohol $\left(90 \mathrm{mg}, 89 \%\right.$ yield, $\mathrm{R}_{f}=0.37$ in $3: 1$ hexanes:EtOAc) as a colorless oil: ${ }^{1} \mathrm{H} \mathrm{NMR}(400 \mathrm{MHz}$, $\left.\mathrm{CDCl}_{3}\right) \square 7.20(\mathrm{~d}, 2 \mathrm{H}, J=8.4 \mathrm{~Hz}), 6.84(\mathrm{~d}, 2 \mathrm{H}, J=8.8 \mathrm{~Hz}), 6.15$ (quintet, $\left.1 \mathrm{H}, J=2.8 \mathrm{~Hz}\right), 3.78(\mathrm{~s}, 3 \mathrm{H}), 3.75(\mathrm{t}, 2 \mathrm{H}$, $J=6.4 \mathrm{~Hz}$ ), 2.39-2.27 (m, 2H), 2.10-2.07 (m, 2H), 1.83 (br s, 1H), 1.50-1.44 (m, 2H), 1.35 (sextet, 2H, $J=7.2 \mathrm{~Hz}$ ), $0.88(\mathrm{t}, 3 \mathrm{H}, J=7.2 \mathrm{~Hz}) ;{ }^{13} \mathrm{C} \mathrm{NMR}\left(100 \mathrm{MHz}, \mathrm{CDCl}_{3}\right) \square 201.1,158.7,127.7,127.5,114.2,105.7,95.6,61.0,55.3$, $36.0,32.9,29.8,22.5,14.0$.<smiles>CC(=Cc1ccc(C(F)(F)F)cc1)CCO</smiles>

The crude mixture was purified by flash column chromatography (9:1 hexanes:EtOAc eluent) to afford the desired homoallenic alcohol $\left(87 \mathrm{mg}, 86 \%\right.$ yield, $\mathrm{R}_{f}=0.12$ in 9:1 hexanes:EtOAc) as a colorless oil: ${ }^{1} \mathrm{H} \mathrm{NMR}(400 \mathrm{MHz}$, $\left.\mathrm{CDCl}_{3}\right) \square 7.33(\mathrm{~d}, 2 \mathrm{H}, J=8.0 \mathrm{~Hz}), 7.07(\mathrm{~d}, 2 \mathrm{H}, J=8.0 \mathrm{~Hz}), 5.87($ sextet, $1 \mathrm{H}, J=2.8 \mathrm{~Hz}), 3.45(\mathrm{t}, 2 \mathrm{H}, J=6.0 \mathrm{~Hz})$, $2.02(\mathrm{bt}, 2 \mathrm{H}, J=6.4 \mathrm{~Hz}), 1.56(\mathrm{~d}, 3 \mathrm{H}, J=2.8 \mathrm{~Hz}), 1.43($ br s, $1 \mathrm{H}) ;{ }^{13} \mathrm{C} \mathrm{NMR}\left(100 \mathrm{MHz}, \mathrm{CDCl}_{3}\right) \square 203.8,139.6$, $127.7(\mathrm{t}, J=)$ ) 126.8, 125.5, 125.4, 101.3, 93.4, 60.2, 36.8, 18.2; HRMS (EI ${ }^{+}$) calc'd for $\left[\mathrm{C}_{13} \mathrm{H}_{13} \mathrm{~F}_{3} \mathrm{O}^{+}: \mathrm{m} / z\right.$ 242.0918, found 242.0919; Anal calc'd for $\mathrm{C}_{13} \mathrm{H}_{13} \mathrm{~F}_{3} \mathrm{O}$ : C, 64.46; H, 5.4; found: C, 64.29; H, 5.58.<smiles>CC(C)(C)C(=CCc1ccccc1Br)CCO</smiles>

5f (Table 1, entry 6)

The crude mixture was purified by flash column chromatography (9:1 hexanes:EtOAc eluent) to afford the desired homoallenic alcohol $\left(97 \mathrm{mg}, 96 \%\right.$ yield, $\mathrm{R}_{f}=0.36$ in 3:1 hexanes:EtOAc) as a colorless oil: ${ }^{1} \mathrm{H} \mathrm{NMR}(400 \mathrm{MHz}$, $\left.\mathrm{CDCl}_{3}\right) \square 7.50(\mathrm{dd}, 1 \mathrm{H}, J=8.0,1.2 \mathrm{~Hz}), 7.42(\mathrm{dd}, 1 \mathrm{H}, J=7.6,1.6 \mathrm{~Hz}), 7.22(\mathrm{td}, 1 \mathrm{H}, J=7.6,1.2 \mathrm{~Hz}), 7.01(\mathrm{td}, 1 \mathrm{H}, J$ $=8.0,1.6 \mathrm{~Hz}$ ), 6.62 (quintet, $1 \mathrm{H}, J=3.2 \mathrm{~Hz}), 3.76(\mathrm{t}, 2 \mathrm{H}, J=6.0 \mathrm{~Hz}), 2.37-2.32(\mathrm{~m}, 2 \mathrm{H}), 2.13-2.08(\mathrm{~m}, 2 \mathrm{H}), 1.68$ (br s, $\left.1 \mathrm{H}), 1.49-1.42(\mathrm{~m}, 2 \mathrm{H}), 1.39-1.31(\mathrm{~m}, 2 \mathrm{H}), 0.88(\mathrm{t}, 3 \mathrm{H}, J=7.6 \mathrm{~Hz}) ;{ }^{13} \mathrm{C} \mathrm{NMR}\left(100 \mathrm{MHz}^{\mathrm{C}} \mathrm{CDCl}\right)_{3}\right) 203.1$, 134.7, 133.1, 128.0 (2), 127.5, 122.4, 106.0, 95.0, 60.8, 35.8, 32.6, 29.7, 22.5, 13.9; HRMS (EI') calc'd for $\left[\mathrm{C}_{15} \mathrm{H}_{19} \mathrm{BrO}\right]^{+}: \mathrm{m} / z$ 294.0619, found 294.0615; Anal calc'd for $\mathrm{C}_{15} \mathrm{H}_{19} \mathrm{BrO}: \mathrm{C}, 61.03 ; \mathrm{H}, 6.49$; found: $\mathrm{C}$, 60.76; $\mathrm{H}$, 6.55 .<smiles>CCCCCC=C=C(CCO)c1ccccc1</smiles>

5g (Table 1, entry 7)

The crude mixture was purified by flash column chromatography (9:1 hexanes:EtOAc eluent) to afford the desired homoallenic alcohol (94 mg, 93\% yield, $\mathrm{R}_{f}=0.15$ in 9:1 hexanes:EtOAc) as a colorless oil: ${ }^{1} \mathrm{H} \mathrm{NMR} \mathrm{(400} \mathrm{MHz,}$ $\left.\mathrm{C}_{6} \mathrm{D}_{6}\right) \square 7.50(\mathrm{~d}, 2 \mathrm{H}, J=7.6 \mathrm{~Hz}), 7.27-7.24(\mathrm{~m}, 2 \mathrm{H}), 7.13(\mathrm{t}, 1 \mathrm{H}, J=7.6 \mathrm{~Hz}), 5.45($ septet, $1 \mathrm{H}, J=2.8 \mathrm{~Hz}), 3.81(\mathrm{t}$, $2 \mathrm{H}, J=6.8 \mathrm{~Hz}), 2.67-2.63(\mathrm{~m}, 2 \mathrm{H}), 2.03(\mathrm{q}, 2 \mathrm{H}, J=7.6 \mathrm{~Hz}), 1.72(\mathrm{br} \mathrm{s}, 1 \mathrm{H}), 1.48-1.40(\mathrm{~m}, 2 \mathrm{H}), 1.30-1.22(\mathrm{~m}, 4 \mathrm{H})$, $0.92(\mathrm{t}, 3 \mathrm{H}, J=6.8 \mathrm{~Hz}) ;{ }^{13} \mathrm{C}$ NMR $\left(100 \mathrm{MHz}, \mathrm{C}_{6} \mathrm{D}_{6}\right) \square 203.8,137.0,128.4,126.6,126.0,102.7,94.8,61.1,33.4$, 31.4, 29.0 (2), 22.5, 14.0; HRMS $\left(\mathrm{EI}^{+}\right)$calc'd for $\left[\mathrm{C}_{16} \mathrm{H}_{22} \mathrm{O}\right]^{+}: \mathrm{m} / z$ 230.1671, found 230.1667; Anal calc'd for $\mathrm{C}_{16} \mathrm{H}_{22} \mathrm{O}: \mathrm{C}, 83.43$; H, 9.63; found: C, 81.69; H, 9.80 . 
<smiles>CC(C)C=C=C(P)CCO</smiles>

5h (Table 1, entry 8 )

The crude mixture was purified by flash column chromatography ( $9: 1$ hexanes:EtOAc eluent) to afford the desired homoallenic alcohol (88 mg, 87\% yield, $\mathrm{R}_{f}=0.20$ in 9:1 hexanes:EtOAc) as a colorless oil: ${ }^{1} \mathrm{H}$ NMR (400 MHz, $\left.\mathrm{C}_{6} \mathrm{D}_{6}\right) \square 7.50(\mathrm{~d}, 2 \mathrm{H}, J=7.2 \mathrm{~Hz}), 7.28-7.23(\mathrm{~m}, 2 \mathrm{H}), 7.13(\mathrm{t}, 1 \mathrm{H}, J=7.2 \mathrm{~Hz}), 5.50-5.46(\mathrm{~m}, 1 \mathrm{H}), 3.79(\mathrm{t}, 2 \mathrm{H}, 6.6$ $\mathrm{Hz}), 2.63(\mathrm{td}, 2 \mathrm{H}, J=6.6,3.6 \mathrm{~Hz}), 2.34-2.27$ (septet, $1 \mathrm{H}, J=6.6 \mathrm{~Hz}), 1.59(\mathrm{br} \mathrm{s}, 1 \mathrm{H}), 1.05(\mathrm{~d}, 6 \mathrm{H}, J=6.6 \mathrm{~Hz}) ;{ }^{13} \mathrm{C}$ NMR (100 MHz, $\left.\mathrm{C}_{6} \mathrm{D}_{6}\right) \square 202.4,137.4,128.7,127.0,126.2,104.3,102.4,61.4,33.8,29.0,22.7$; HRMS (EI $\left.{ }^{+}\right)$calc'd for $\left[\mathrm{C}_{14} \mathrm{H}_{18} \mathrm{O}\right]^{+}: m / z 202.1358$, found 202.1359 .

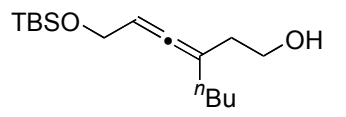

5i (Table 1, entry 9)

The crude mixture was purified by flash column chromatography (9:1 hexanes:EtOAc eluent) to afford the desired homoallenic alcohol (77 mg, 76\% yield, $\mathrm{R}_{f}=0.42$ in 9:1 hexanes:EtOAc) as a colorless oil: ${ }^{1} \mathrm{H}$ NMR $(400 \mathrm{MHz}$, $\left.\mathrm{CDCl}_{3}\right) \square$ 5.29-5.24 (m, 1H), 4.19-4.09 (m, 2H), 3.76-3.70 (m, 1H), 3.65-3.59 (m, 1H), 2.25-2.17 (m, 1H), 2.16-2.08 $(\mathrm{m}, 1 \mathrm{H}), 1.97-1.89(\mathrm{~m}, 2 \mathrm{H}), 1.45-1.26(\mathrm{~m}, 4 \mathrm{H}), 0.88(\mathrm{t}, 3 \mathrm{H}, J=7.2 \mathrm{~Hz}), 0.87(\mathrm{~s}, 9 \mathrm{H}), 0.06(\mathrm{~s}, 6 \mathrm{H}) ;{ }^{13} \mathrm{C}$ NMR $(100$ $\left.\mathrm{MHz}, \mathrm{CDCl}_{3}\right) \square 199.5,104.4,93.4,60.9,60.0,35.7,32.6,29.7,25.8,22.4,18.4,14.0,-5.4$; HRMS (FAB) calc'd for $\left[\mathrm{C}_{16} \mathrm{H}_{32} \mathrm{O}_{2} \mathrm{SiLi}\right]^{+}: \mathrm{m} / z$ 291.2332, found 291.2327; Anal calc'd for $\mathrm{C}_{16} \mathrm{H}_{32} \mathrm{O}_{2} \mathrm{Si}$ : C, 67.54; H, 11.34; found: C, 65.58; $\mathrm{H}$, 10.78 .<smiles>CC=C=C(CCO)CCCc1ccccc1</smiles>

\section{$\mathbf{5 j}$ (Table 1, entry 10)}

The crude mixture was purified by flash column chromatography (10:1 hexanes:EtOAc eluent) to afford the desired homoallenic alcohol ( $84 \mathrm{mg}, 84 \%$ yield, $\mathrm{R}_{f}=0.40$ in $3: 1$ hexanes:EtOAc) as a colorless oil: ${ }^{1} \mathrm{H}$ NMR (400 MHz, $\left.\mathrm{CDCl}_{3}\right) \square 7.31-7.27(\mathrm{~m}, 2 \mathrm{H}), 7.21-7.18(\mathrm{~m}, 3 \mathrm{H}), 5.22-5.15(\mathrm{~m}, 1 \mathrm{H}), 3.72(\mathrm{t}, 2 \mathrm{H}, J=6.0 \mathrm{~Hz}), 2.65(\mathrm{t}, 2 \mathrm{H}, J=7.6 \mathrm{~Hz})$, 2.23-2.18 (m, 2H), $2.00(\mathrm{td}, 2 \mathrm{H}, J=7.2,2.8 \mathrm{~Hz}), 1.86(\mathrm{br} \mathrm{s}, 1 \mathrm{H}), 1.76$ (quintet, $2 \mathrm{H}, J=7.6 \mathrm{~Hz}$ ), $1.68(\mathrm{~d}, 3 \mathrm{H}, J=6.8$ $\mathrm{Hz}) ;{ }^{13} \mathrm{C}$ NMR $\left(100 \mathrm{MHz}, \mathrm{CDCl}_{3}\right) \square 201.5,142.5,128.5,128.3,125.8,100.3,87.7,60.9,35.9,35.5,32.3,29.3,15.1$; HRMS $\left(\mathrm{EI}^{+}\right)$calc'd for $\left[\mathrm{C}_{15} \mathrm{H}_{20} \mathrm{O}\right]^{+}: m / z$ 216.1514, found 216.1513; Anal calc'd for $\mathrm{C}_{15} \mathrm{H}_{20} \mathrm{O}: \mathrm{C}, 83.28 ; \mathrm{H}, 9.32$; found: $\mathrm{C}, 83.0 ; \mathrm{H}, 9.60$.<smiles></smiles>

5k (Table 1, entry 11)

The crude mixture was purified by flash column chromatography ( $9: 1$ hexanes:EtOAc eluent) to afford the desired homoallenic alcohol (91 mg, 90\% yield, $\mathrm{R}_{f}=0.24$ in 9:1 hexanes:EtOAc) as a colorless oil: ${ }^{1} \mathrm{H}$ NMR (400 MHz, $\left.\mathrm{CDCl}_{3}\right) \square 5.20-5.16(\mathrm{~m}, 1 \mathrm{H}), 3.72(\mathrm{t}, 2 \mathrm{H}, J=6.4 \mathrm{~Hz}), 2.30-2.26(\mathrm{~m}, 2 \mathrm{H}), 1.92(\mathrm{q}, 2 \mathrm{H}, J=7.6 \mathrm{~Hz}), 1.80(\mathrm{~s}, 1 \mathrm{H}), 1.37-$ $1.26(\mathrm{~m}, 6 \mathrm{H}), 1.10-1.06(\mathrm{~m}, 1 \mathrm{H}), 0.86(\mathrm{t}, 3 \mathrm{H}, J=6.4 \mathrm{~Hz}), 0.64-0.60(\mathrm{~m}, 2 \mathrm{H}), 0.38-0.31(\mathrm{~m}, 2 \mathrm{H}) ;{ }^{13} \mathrm{C} \mathrm{NMR}(100$ $\left.\mathrm{MHz}, \mathrm{CDCl}_{3}\right) \square$ 199.3, 104.1, 94.2, 61.0, 36.0, 31.3, 29.4, 28.9, 22.4, 14.0, 12.6, 6.7, 6.2; HRMS (EI ${ }^{+}$) calc'd for $\left[\mathrm{C}_{13} \mathrm{H}_{22} \mathrm{O}\right]^{+}: \mathrm{m} / z$ 194.1671, found 194.1669.<smiles>CC(CCO)CCc1ccccc1</smiles>

5l (Table 1, entry 12)

The crude mixture was purified by flash column chromatography ( $9: 1$ hexanes:EtOAc eluent) to afford the desired homoallenic alcohol (92 mg, 91\% yield, $\mathrm{R}_{f}=0.36$ in 3:1 hexanes:EtOAc) as a colorless oil: ${ }^{1} \mathrm{H}$ NMR (400 MHz, $\left.\mathrm{CDCl}_{3}\right) \square 7.30-7.25(\mathrm{~m}, 2 \mathrm{H}), 7.20-7.16(\mathrm{~m}, 3 \mathrm{H}), 3.60(\mathrm{t}, 2 \mathrm{H}, J=6.4 \mathrm{~Hz}), 2.72(\mathrm{t}, 2 \mathrm{H}, J=7.6 \mathrm{~Hz}), 2.27(\mathrm{t}, 2 \mathrm{H}, J=8.4$ $\mathrm{Hz}), 2.12(\mathrm{t}, 3 \mathrm{H}, J=6.4 \mathrm{~Hz}), 1.71(\mathrm{~s}, 3 \mathrm{H}), 1.64(\mathrm{br} \mathrm{s}, 1 \mathrm{H}), 1.61(\mathrm{~s}, 3 \mathrm{H}) ;{ }^{13} \mathrm{C}$ NMR $\left(100 \mathrm{MHz}, \mathrm{CDCl}_{3}\right) \square 198.8,142.1$, 128.4, 128.3, 125.8, 99.0, 95.9, 60.9, 37.4, 36.0, 34.0, 19.6 (2); Anal calc'd for $\mathrm{C}_{15} \mathrm{H}_{20} \mathrm{O}: \mathrm{C}, 83.28 ; \mathrm{H}, 9.32$; found: $\mathrm{C}$, 83.02; H, 9.05 . 


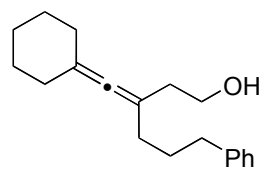

$\mathbf{5 m}$ (Table 1, entry 13)

The crude mixture was purified by flash column chromatography (9:1 hexanes:EtOAc eluent) to afford the desired homoallenic alcohol (31 mg, 61\% yield, $\mathrm{R}_{f}=0.13$ in 9:1 hexanes:EtOAc) as a colorless oil: ${ }^{1} \mathrm{H} \mathrm{NMR}(400 \mathrm{MHz}$, $\left.\mathrm{CDCl}_{3}\right) \square 7.29-7.24(\mathrm{~m}, 2 \mathrm{H}), 7.18-7.16(\mathrm{~m}, 3 \mathrm{H}), 3.70(\mathrm{t}, 2 \mathrm{H}, J=6.0 \mathrm{~Hz}), 2.62(\mathrm{t}, 2 \mathrm{H}, J=7.6 \mathrm{~Hz}), 2.17(\mathrm{t}, 2 \mathrm{H}, J=6.0$ $\mathrm{Hz}), 2.11(\mathrm{t}, 4 \mathrm{H}, J=5.6 \mathrm{~Hz}), 1.96(\mathrm{t}, 2 \mathrm{H}, J=7.2 \mathrm{~Hz}), 1.73$ (quintet, $2 \mathrm{H}, J=7.2 \mathrm{~Hz}), 1.64-1.49(\mathrm{~m}, 7 \mathrm{H}) ;{ }^{13} \mathrm{C} \mathrm{NMR}$ $\left(100 \mathrm{MHz}, \mathrm{CDCl}_{3}\right) \square 194.6,142.6,128.5,128.3,125.7,104.9,98.4,61.1,36.3,35.3,32.6,32.2,29.3,27.8,26.2$; HRMS $\left(\mathrm{EI}^{+}\right)$calc'd for $\left[\mathrm{C}_{19} \mathrm{H}_{26} \mathrm{O}\right]^{+}: \mathrm{m} / z$ 270.1984, found 270.1983; Anal calc'd for $\mathrm{C}_{19} \mathrm{H}_{26} \mathrm{O}: \mathrm{C}, 84.39$; $\mathrm{H}, 9.69$; found: C, 84.91; H, 9.68.

\section{Determination of Allene Absolute Stereochemistry:}<smiles>C#CC(OC=C)c1ccccc1</smiles>

(R)-4a

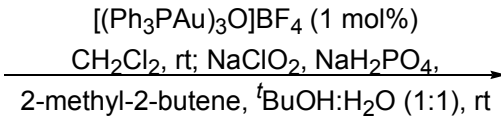

2-methyl-2-butene, ${ }^{\mathrm{B}} \mathrm{BuOH}: \mathrm{H}_{2} \mathrm{O}(1: 1)$, rt

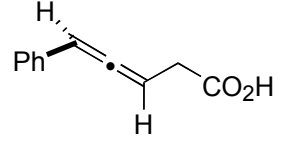

(R)-15

To a solution of $(\mathbf{R})-4 \mathbf{a}(0.100 \mathrm{~g}, 0.632 \mathrm{mmol}, 92 \%$ ee $)$ in $\mathrm{CH}_{2} \mathrm{Cl}_{2}(1.8 \mathrm{~mL})$ was added $\left[\left(\mathrm{Ph}_{3} \mathrm{PAu}\right) \mathrm{O}\right] \mathrm{BF} 4(9.3 \mathrm{mg}, 6.3$ $\square \mathrm{mol}$ ) and the resulting mixture was left to stir at $\mathrm{rt}$ for $3 \mathrm{~h}$. The reaction mixture was concentrated, the residue dissolved in $t$ - $\mathrm{BuOH}(6 \mathrm{~mL})$, and 2-methyl-2-butene $(1.8 \mathrm{~mL})$ was added. To the resulting yellow mixture was added a solution of $\mathrm{NaClO}_{2}(85 \mathrm{mg}, 0.95 \mathrm{mmol})$ and $\mathrm{NaH}_{2} \mathrm{PO}_{4}(131 \mathrm{mg}, 0.948 \mathrm{mmol})$ in $\mathrm{H}_{2} \mathrm{O}(6 \mathrm{~mL})$, and the biphasic mixture was left to stir at $\mathrm{rt}$ for $4 \mathrm{~h}$. Organic solvents were removed in vacuo, the resulting mixture was diluted with $\mathrm{H}_{2} \mathrm{O}(30 \mathrm{~mL})$, and extracted with hexanes $(3 \mathrm{x}, 30 \mathrm{~mL})$. The aqueous layer was cooled to $0{ }^{\circ} \mathrm{C}$ and acidified to $\mathrm{pH} 1$ by dropwise addition of concentrated $\mathrm{HCl}$. Organics were extracted with EtOAc $(6 \mathrm{x}, 30 \mathrm{~mL})$, the combined extracts were dried over $\mathrm{MgSO}_{4}$, filtered, and concentrated to afford a pale yellow liquid. The crude residue was purified by flash column chromatography (1\% AcOH in 5:1 hexanes:EtOAc eluent) to afford the desired carboxylic acid (R)-15 (22 mg, 66\% yield, $\mathrm{R}_{f}=0.13$ in 3:1 hexanes:EtOAc) as a colorless oil: Spectral data were consistent with those previously reported; ${ }^{7}[\square]_{\mathrm{D}}=-31\left(\mathrm{c} 1.0, \mathrm{CHCl}_{3}\right),\left(\right.$ lit: $\left.{ }^{7}[\square]_{\mathrm{D}}=+102.2\left(\mathrm{c} 2.0, \mathrm{CHCl}_{3}\right)\right)$.

\section{Allene Racemization Experiments:}

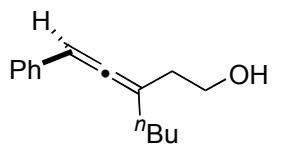

(R)-2
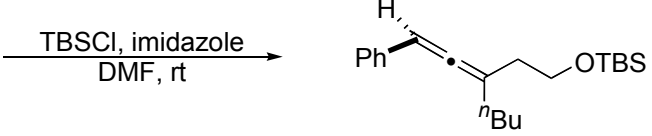

(R)-14

To a solution of (R)-2 $(85 \mathrm{mg}, 0.39 \mathrm{mmol})$ in DMF $(0.5 \mathrm{~mL})$ was added imidazole $(59 \mathrm{mg}, 0.87 \mathrm{mmol})$ followed by TBSCl (71 mg, $0.47 \mathrm{mmol}$ ) and the resulting mixture was left to stir at $\mathrm{rt}$ for $3 \mathrm{~d}$. The crude mixture was purified directly by flash column chromatography (50:1 hexanes:EtOAc eluent) to afford the desired silyl ether (R)-14 (118 mg, 91\% yield, $\mathrm{R}_{f}=0.88$ in 3:1 hexanes:EtOAc) as a colorless oil: ${ }^{1} \mathrm{H}$ NMR $\left(400 \mathrm{MHz}, \mathrm{CDCl}_{3}\right) \square 7.28-7.25$ (m, 4H), 7.17-7.15 (m, 1H), $6.10(\mathrm{t}, 1 \mathrm{H}, J=2.8 \mathrm{~Hz}), 3.74(\mathrm{t}, 2 \mathrm{H}, J=7.2 \mathrm{~Hz}), 2.31(\mathrm{t}, 2 \mathrm{H}, J=7.2 \mathrm{~Hz}), 2.11-2.08(\mathrm{~m}, 2 \mathrm{H})$, $1.47-1.43(\mathrm{~m}, 2 \mathrm{H}), 1.37-1.33(\mathrm{~m}, 2 \mathrm{H}), 0.89-0.86(\mathrm{~m}, 12 \mathrm{H}), 0.03(\mathrm{~s}, 6 \mathrm{H}) ;{ }^{13} \mathrm{C} \mathrm{NMR}\left(100 \mathrm{MHz}^{\mathrm{CDCl}} \mathrm{CD}_{3}\right) \square 2.5$, $136.0,128.5,126.5,126.4,105.6,95.3,62.0,36.1,32.8,29.8,25.8,22.5,18.3,14.0,-5.2$.<smiles>CC(C)(CC=Cc1ccccc1)CCO[SbH2]</smiles>

(R)-14
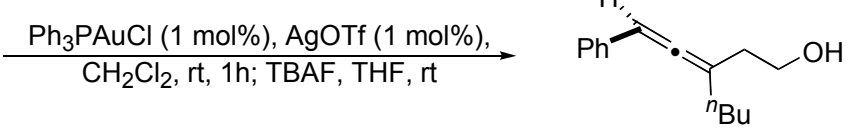

(R)-2

To a solution of (R)-14 (42.2 mg, $0.128 \mathrm{mmol}, 83 \%$ ee) in $\mathrm{CH}_{2} \mathrm{Cl}_{2}(0.36 \mathrm{~mL})$ was added $\mathrm{Ph}_{3} \mathrm{PAuCl}(0.6 \mathrm{mg}, 1.3$ $\square \mathrm{mol})$ followed by AgOTf $(0.2 \mathrm{mg}, 1.3 \square \mathrm{mol})$ and the cloudy mixture was left to stir at $\mathrm{rt}$ for $1 \mathrm{~h}$. The resulting 
solution was filtered through silica gel washing with $\mathrm{CH}_{2} \mathrm{Cl}_{2}(2 \mathrm{~mL})$ and the solvent removed to afford a yellow oil. The oil was dissolved in THF $(0.36 \mathrm{~mL})$, TBAF $(0.15 \mathrm{~mL}, 0.15 \mathrm{mmol}, 1.0 \mathrm{M}$ in THF $)$ was added, and the reaction mixture was left to stir at $\mathrm{rt}$ for $10 \mathrm{~min}$. The crude mixture was purified directly by flash column chromatography (9:1 hexanes:EtOAc eluent) to afford the desired homoallenic alcohol $\left(19 \mathrm{mg}, 68 \%\right.$ yield, $\mathrm{R}_{f}=0.14$ in 9:1 hexanes:EtOAc) as a colorless oil: Spectral data were consistent with those described for 2; Enantiomeric purity was determined by chiral HPLC analysis, Chiralcel OD column, 97:3 hexanes:isopropanol, $1 \mathrm{~mL} / \mathrm{min} ; \mathrm{t}_{\mathrm{R}} 9.14 \mathrm{~min}$ (minor), 12.49 min (major): 4\% ee.

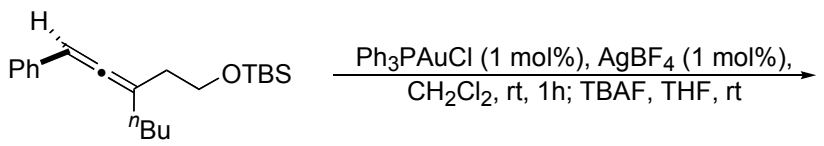

(R)-14

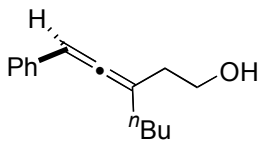

(R)-2

To a suspension of $\mathrm{AgBF}_{4}(0.3 \mathrm{mg}, 1.5 \square \mathrm{mol})$ in $\mathrm{CH}_{2} \mathrm{Cl}_{2}(0.2 \mathrm{~mL})$ was added $\mathrm{Ph}_{3} \mathrm{PAuCl}(0.7 \mathrm{mg}, 1.5 \square \mathrm{mol})$ and the resulting cloudy solution was stirred rapidly at rt for $5 \mathrm{~min}$. The mixture was filtered through a cotton plug into a solution of $(\mathbf{R})-14\left(50.0 \mathrm{mg}, 0.151 \mathrm{mmol}, 90 \%\right.$ ee) in $\mathrm{CH}_{2} \mathrm{Cl}_{2}(0.4 \mathrm{~mL})$ and the reaction mixture was left to stir at $\mathrm{rt}$ for $1 \mathrm{~h}$. The solution was filtered through a silica gel plug washing with 4:1 hexanes:EtOAc and the solvent removed to afford a yellow oil. The resulting oil was dissolved in THF $(0.43 \mathrm{~mL})$, TBAF $(0.18 \mathrm{~mL}, 0.18 \mathrm{mmol}, 1.0$ $\mathrm{M}$ in THF) was added, and the reaction mixture was left to stir at $\mathrm{rt}$ for $1 \mathrm{~h}$. The crude mixture was purified directly by flash column chromatography (9:1 hexanes:EtOAc eluent) to afford the desired homoallenic alcohol $(27 \mathrm{mg}$, $83 \%$ yield, $\mathrm{R}_{f}=0.14$ in 9:1 hexanes:EtOAc) as a colorless oil: Spectral data were consistent with those described for 2; Enantiomeric purity was determined by chiral HPLC analysis, Chiralcel OD column, 97:3 hexanes:isopropanol, 1 $\mathrm{mL} / \mathrm{min} ; \mathrm{t}_{\mathrm{R}} 14.98 \mathrm{~min}, 26.87 \mathrm{~min}: 0 \%$ ee.

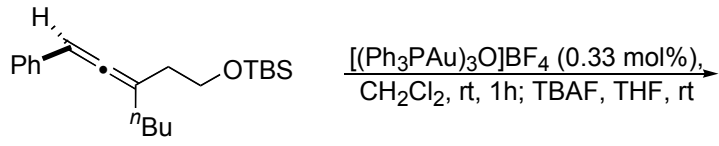

(R)-14

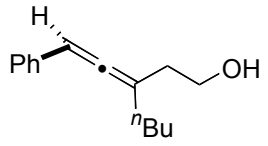

(R)-2

To a solution of $(\mathbf{R})-\mathbf{1 4}(50.0 \mathrm{mg}, 0.151 \mathrm{mmol}, 90 \%$ ee $)$ in $\mathrm{CH}_{2} \mathrm{Cl}_{2}(0.43 \mathrm{~mL})$ was added $\left.\left[\left(\mathrm{Ph}_{3} \mathrm{PAu}\right)_{3} \mathrm{O}\right)\right] \mathrm{BF}_{4}(0.7 \mathrm{mg}$, $0.5 \square \mathrm{mol}$ ) and the resulting solution was left to stir at $\mathrm{rt}$ for $1 \mathrm{~h}$. The reaction mixture was filtered through a silica gel plug washing with 4:1 hexanes:EtOAc and the solution concentrated to afford a yellow oil. The resulting oil was dissolved in THF $(0.43 \mathrm{~mL})$, TBAF $(0.18 \mathrm{~mL}, 0.18 \mathrm{mmol}, 1.0 \mathrm{M}$ in THF) was added, and the reaction mixture was left to stir at $\mathrm{rt}$ for $1 \mathrm{~h}$. The crude mixture was purified directly by flash column chromatography $(9: 1$ hexanes:EtOAc eluent) to afford the desired homoallenic alcohol $\left(31 \mathrm{mg}, 95 \%\right.$ yield, $\mathrm{R}_{f}=0.14$ in 9:1 hexanes:EtOAc) as a colorless oil: Spectral data were consistent with those described for 2; Enantiomeric purity was determined by chrial HPLC analysis, Chiralcel OD column, 97:3 hexanes:isopropanol, $1 \mathrm{~mL} / \mathrm{min} ; \mathrm{t}_{\mathrm{R}} 14.34 \mathrm{~min}$ (minor), $26.59 \min$ (major): 67\% ee.

\section{Chirality Transfer Experiments:}<smiles>OCC/C=C/c1ccccc1</smiles>

\section{(R)-5a}

The Claisen rearrangement was carried out following the general procedure described above using (R)-4a of $92 \%$ ee. The crude mixture was purified by flash column chromatography ( $7: 1$ hexanes:EtOAc eluent) to afford the desired homoallenic alcohol (39 mg, 78\% yield, $\mathrm{R}_{f}=0.21$ in 3:1 hexanes:EtOAc) as a pale yellow oil: Spectral data were consistent with those previously described for 5a; Enantiomeric purity was determined by chiral HPLC analysis, Chiralcel OD column, 97:3 hexanes:isopropanol, $1 \mathrm{~mL} / \mathrm{min}$; $t_{\mathrm{R}} 21.42 \mathrm{~min}$ (minor), $24.43 \mathrm{~min}$ (major): 88\% ee. 
<smiles>CC=C(CCO)CCc1ccccc1</smiles>

(R)-5j

The Claisen rearrangement was carried out following the general procedure described above using (R)-4j of $79 \%$ ee. The crude mixture was purified by flash column chromatography (10:1 hexanes:EtOAc eluent) to afford the desired homoallenic alcohol (84 mg, 84\% yield, $\mathrm{R}_{f}=0.36$ in 3:1 hexanes:EtOAc) as a colorless oil: Spectral data were consistent with those described for $\mathbf{5} \mathbf{j}$; Enantiomeric purity was determined by chiral HPLC analysis, Chiralcel OD column, 99.2:0.8 hexanes:isopropanol, $1 \mathrm{~mL} / \mathrm{min} ; \mathrm{t}_{\mathrm{R}} 33.23 \mathrm{~min}$ (major), $35.17 \mathrm{~min}$ (minor): $77 \%$ ee.

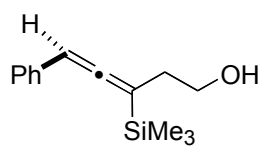

\section{(S)-5n}

The Claisen rearrangement was carried out following the general procedure described above using (R)-5n of $92 \%$ ee. The crude mixture was purified by flash column chromatography (9:1 hexanes:EtOAc eluent) to afford the desired homoallenic alcohol (49 mg, $98 \%$ yield, $\mathrm{R}_{f}=0.40$ in 3:1 hexanes:EtOAc) as a colorless oil: ${ }^{1} \mathrm{H}$ NMR (400 $\left.\mathrm{MHz}, \mathrm{CDCl}_{3}\right)$ ] 7.35-7.26 (m, 4H), 7.21-7.17 (m, 1H), 5.99 (t, 1H, $\left.J=3.2 \mathrm{~Hz}\right), 3.84(\mathrm{t}, 2 \mathrm{H}, J=6.4 \mathrm{~Hz}), 2.46-2.39$ (m, 2H), 1.79 (br s, $1 \mathrm{H}), 0.22$ (s, 9H); ${ }^{13} \mathrm{C} \mathrm{NMR}\left(100 \mathrm{MHz}, \mathrm{CDCl}_{3}\right) \square 205.0,135.4,128.7,126.2,125.9,98.1,90.3$, 62.2, 32.5, -1.4; HRMS $\left(\mathrm{EI}^{+}\right)$calc'd for $\left[\mathrm{C}_{14} \mathrm{H}_{20} \mathrm{OSi}\right]^{+}: \mathrm{m} / z$ 232.1283, found 232.1281; Anal calc'd for $\mathrm{C}_{14} \mathrm{H}_{20} \mathrm{OSi}$ : C, 72.36; H, 8.67; found: C, 72.78; H, 8.71; Enantiomeric purity was determined by chiral HPLC analysis, Chiralcel OD column, 97:3 hexanes:isopropanol, $1 \mathrm{~mL} / \mathrm{min}$; $t_{\mathrm{R}} 9.99 \min$ (minor), $23.12 \mathrm{~min}$ (major): $92 \%$ ee.

\section{Diastereoselectivity Experiments:}
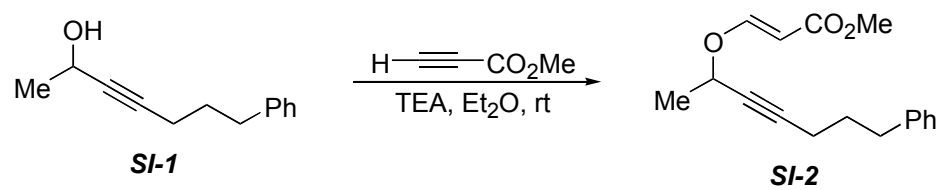

The preparation of $\mathbf{S I - 2}$ was carried out according to the method of Ireland and Wipf. ${ }^{8}$ The crude red oil was purified by flash column chromatography (15:1 hexanes:EtOAc eluent) to afford the desired vinylogous carbonate SI-2 (1.84 g, 64\% yield, $\mathrm{R}_{f}=0.58$ in 3:1 hexanes:EtOAc) as a colorless liquid: ${ }^{1} \mathrm{H}$ NMR $\left(400 \mathrm{MHz}, \mathrm{CDCl}_{3}\right) \square 7.64$ $(\mathrm{d}, 1 \mathrm{H}, J=12.8 \mathrm{~Hz}), 7.27$ (t, $2 \mathrm{H}, J=7.6 \mathrm{~Hz}), 7.18(\mathrm{t}, 3 \mathrm{H}, J=8.0 \mathrm{~Hz}), 5.38(\mathrm{~d}, 1 \mathrm{H}, J=12.4 \mathrm{~Hz}), 4.68($ br q, $1 \mathrm{H}, J=$ $6.4 \mathrm{~Hz}$ ), $3.68(\mathrm{~s}, 3 \mathrm{H}), 2.69(\mathrm{t}, 2 \mathrm{H}, J=7.6 \mathrm{~Hz}), 2.23(\mathrm{td}, 2 \mathrm{H}, J=6.8,1.6 \mathrm{~Hz}), 1.82$ (quintet, $2 \mathrm{H}, J=7.2 \mathrm{~Hz}), 1.54(\mathrm{~d}$, $3 \mathrm{H}, J=6.8 \mathrm{~Hz}) ;{ }^{13} \mathrm{C}$ NMR $\left(100 \mathrm{MHz}, \mathrm{CDCl}_{3}\right) \square 168.2,160.5,141.4,128.5,128.4,126.0,98.3,87.9,78.2,68.0$, 51.1, 34.7, 30.0, 22.2, 18.1; HRMS $\left(\mathrm{EI}^{+}\right)$calc'd for $\left[\mathrm{C}_{17} \mathrm{H}_{20} \mathrm{O}_{3}\right]^{+}: \mathrm{m} / z$ 272.1412, found 272.1409; Anal calc'd for $\mathrm{C}_{17} \mathrm{H}_{20} \mathrm{O}_{3}$ : C, 74.97; H, 7.40; found: $\mathrm{C}, 71.46 ; \mathrm{H}, 7.14$.
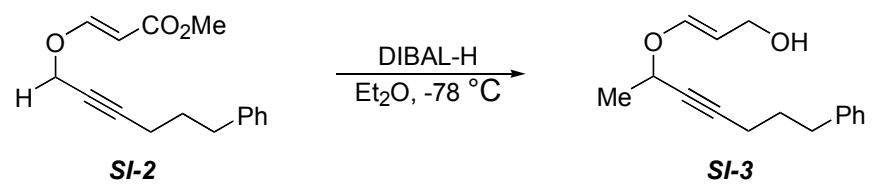

The reduction of $\boldsymbol{S I - 2}$ was carried out according to the method of Ireland and Wipf. ${ }^{8}$ The crude oil was purified by flash column chromatography (2\% TEA in 4:1 hexanes:EtOAc eluent) to afford the desired alcohol SI-3 (0.994 g, $62 \%$ yield, $\mathrm{R}_{f}=0.20$ in 3:1 hexanes:EtOAc) as a colorless oil and the propargyl alcohol $\boldsymbol{S I - 1}\left(0.160 \mathrm{~g}, 13 \%\right.$ yield, $\mathrm{R}_{f}$ $=0.38$ in 3:1 hexanes:EtOAc) as a colorless oil. Characterization for $\boldsymbol{S I}-3:{ }^{1} \mathrm{H}$ NMR $\left(400 \mathrm{MHz}, \mathrm{CDCl}_{3}\right) \square 7.28(\mathrm{t}$, $2 \mathrm{H}, J=8.0 \mathrm{~Hz}), 7.20-7.16(\mathrm{~m}, 2 \mathrm{H}), 6.51(\mathrm{~d}, 1 \mathrm{H}, J=12.8 \mathrm{~Hz}), 5.24(\mathrm{dt}, 1 \mathrm{H}, J=12.4,7.6 \mathrm{~Hz}), 4.53($ br q, $1 \mathrm{H}, J=6.8$ $\mathrm{Hz}$ ), $4.05(\mathrm{~d}, 2 \mathrm{H}, J=7.2 \mathrm{~Hz}), 2.70(\mathrm{t}, 2 \mathrm{H}, J=7.6 \mathrm{~Hz}), 2.22$ (td, $2 \mathrm{H}, J=6.8,1.6 \mathrm{~Hz}), 1.82$ (quintet, $2 \mathrm{H}, J=7.2 \mathrm{~Hz}$ ), 1.49 (d, $3 \mathrm{H}, J=6.4 \mathrm{~Hz}), 1.23$ (br s, $1 \mathrm{H}) ;{ }^{13} \mathrm{C}$ NMR $\left(100 \mathrm{MHz}, \mathrm{CDCl}_{3}\right) \square 147.9,141.5,128.5,128.4,126.0,105.6$, 86.4, 79.5, 66.0, 60.7, 34.7, 30.1, 22.3, 18.1; HRMS $\left(\mathrm{EI}^{+}\right)$calc'd for $\left[\mathrm{C}_{16} \mathrm{H}_{20} \mathrm{O}_{2}-\mathrm{C}_{3} \mathrm{H}_{4} \mathrm{O}\right]^{+}: \mathrm{m} / z$ 188.1201, found 188.1200; Anal calc'd for $\mathrm{C}_{16} \mathrm{H}_{20} \mathrm{O}_{2}:$ C, 78.65; H, 8.25; found: C, 78.96; H, 8.40. 

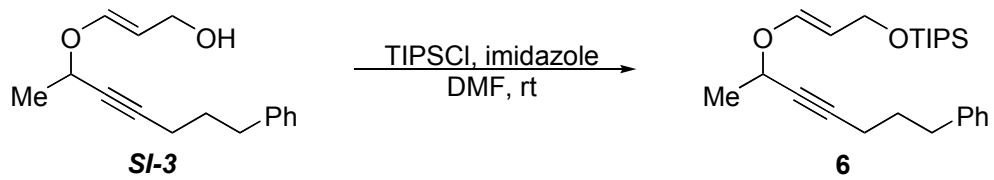

To a solution of $\boldsymbol{S I - 3}(0.946 \mathrm{~g}, 3.87 \mathrm{mmol})$ in DMF $(5.5 \mathrm{~mL})$ was added imidazole $(0.605 \mathrm{~g}, 8.90 \mathrm{mmol})$ followed by TIPSCl $(0.99 \mathrm{~mL}, 4.64 \mathrm{mmol})$, and the resulting mixture was left to stir at rt. TLC analysis after $3 \mathrm{~h}$ indicated consumption of the starting alcohol and the reaction mixture was diluted with $\mathrm{H}_{2} \mathrm{O}(15 \mathrm{~mL})$. The solution was extracted with $\mathrm{Et}_{2} \mathrm{O}(3 \times 10 \mathrm{~mL})$, and the combined organic layers were washed with brine $(3 \mathrm{x} 10 \mathrm{~mL})$, dried over $\mathrm{MgSO}_{4}$, filtered, and concentrated. The crude residue was purified by flash column chromatography $(2 \%$ TEA in hexanes eluent) to afford the desired silyl ether 6 (1.43 g, 92\% yield, $\mathrm{R}_{f}=0.76$ in 3:1 hexanes:EtOAc) as a colorless liquid: ${ }^{1} \mathrm{H}$ NMR $\left(400 \mathrm{MHz}, \mathrm{CDCl}_{3}\right) \square 7.29-7.25(\mathrm{~m}, 2 \mathrm{H}), 7.20-7.16(\mathrm{~m}, 3 \mathrm{H}), 6.47(\mathrm{~d}, 1 \mathrm{H}, J=12.4 \mathrm{~Hz}), 5.16(\mathrm{dt}, 1 \mathrm{H}$, $J=12.4,6.0 \mathrm{~Hz}), 4.51$ (br q, $1 \mathrm{H}, J=6.4 \mathrm{~Hz}), 4.18(\mathrm{~d}, 2 \mathrm{H}, J=6.4 \mathrm{~Hz}), 2.70(\mathrm{t}, 2 \mathrm{H}, J=7.6 \mathrm{~Hz}), 2.21(\mathrm{td}, 2 \mathrm{H}, J=7.2$, $1.6 \mathrm{~Hz}$ ), 1.82 (quintet, $2 \mathrm{H}, J=7.2 \mathrm{~Hz}), 1.48(\mathrm{~d}, 3 \mathrm{H}, J=6.8 \mathrm{~Hz}), 1.12-1.02(\mathrm{~m}, 21 \mathrm{H}) ;{ }^{13} \mathrm{C} \mathrm{NMR}\left(100 \mathrm{MHz}^{\mathrm{CDCl}}{ }_{3}\right) \square$ 145.9, 141.6, 128.5, 128.5, 125.9, 106.3, 86.0, 79.7, 65.8, 61.2, 34.8, 30.2, 22.3, 18.1 (2), 12.1; HRMS (EI $\left.{ }^{+}\right)$calc'd for $\left[\mathrm{C}_{25} \mathrm{H}_{40} \mathrm{O}_{2} \mathrm{Si}\right]^{+}: \mathrm{m} / z$ 400.2797, found 400.2795; Anal calc'd for $\mathrm{C}_{25} \mathrm{H}_{40} \mathrm{O}_{2} \mathrm{Si}$ : C, 74.94; H, 10.06; found: C, 74.68; $\mathrm{H}, 10.07$.

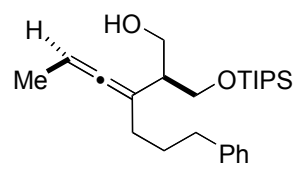

7

The Claisen rearrangement was carried out at $40{ }^{\circ} \mathrm{C}$ following the general procedure described above. The crude mixture was purified by flash column chromatography (14:1 hexanes:EtOAc) to afford unreacted 6 (39 mg, $15 \%$ yield, $\mathrm{R}_{f}=0.71$ in 9:1 hexanes:EtOAc) and the desired homoallenic alcohol 7 (191 mg, 76\% yield, $\mathrm{R}_{f}=0.39$ in 9:1 hexanes:EtOAc, $>20: 1 \mathrm{dr})$ as a colorless oil: ${ }^{1} \mathrm{H}$ NMR $\left(400 \mathrm{MHz}, \mathrm{CDCl}_{3}\right) \square 7.29-7.25(\mathrm{~m}, 2 \mathrm{H}), 7.20-7.16(\mathrm{~m}, 3 \mathrm{H})$, 5.17-5.15 (m, 1H), $3.90(\mathrm{dd}, 1 \mathrm{H}, J=9.6,4.0 \mathrm{~Hz}), 3.78-3.70(\mathrm{~m}, 3 \mathrm{H}), 2.79(\mathrm{br} \mathrm{s}, 1 \mathrm{H}), 2.63(\mathrm{t}, 2 \mathrm{H}, J=7.6 \mathrm{~Hz}), 2.31-$ $2.27(\mathrm{~m}, 1 \mathrm{H}), 2.02-1.99(\mathrm{~m}, 2 \mathrm{H}), 1.73$ (quintet, $2 \mathrm{H}, J=7.6 \mathrm{~Hz}), 1.64(\mathrm{~d}, 3 \mathrm{H}, J=6.8 \mathrm{~Hz}), 1.14-1.03(\mathrm{~m}, 21 \mathrm{H}) ;{ }^{13} \mathrm{C}$ NMR $\left(100 \mathrm{MHz}, \mathrm{CDCl}_{3}\right) \square$ 201.8, 142.4, 128.5, 128.3, 125.7, 101.0, 88.4, 67.2, 66.0, 46.1, 35.4, 31.7, 29.4, 18.0, 15.0, 12.1; HRMS $\left(\mathrm{EI}^{+}\right)$calc'd for $\left[\mathrm{C}_{25} \mathrm{H}_{42} \mathrm{O}_{2} \mathrm{Si}-\mathrm{C}_{3} \mathrm{H}_{6}\right]^{+}: \mathrm{m} / z$ 360.2485, found 360.2428; Anal calc'd for $\mathrm{C}_{25} \mathrm{H}_{42} \mathrm{O}_{2} \mathrm{Si}_{\text {: }}$ C, 74.57; H, 10.51; found: C, 74.73; H, 10.66.

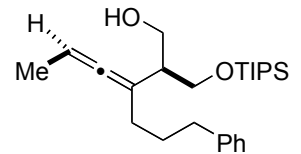

7

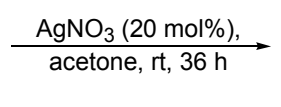

$\underset{\text { acetone, } \mathrm{rt}, 36 \mathrm{~h}}{\stackrel{\mathrm{hNO}}{3}}$

16

The Ag(I)-catalyzed cyclization was carried out using a slight modification of the literature procedure. ${ }^{7}$ To a solution of homoallenic alcohol $7(39 \mathrm{mg}, 98 \square \mathrm{mol})$ in acetone $(0.9 \mathrm{~mL})$ was added $\mathrm{AgNO}_{3}(1.6 \mathrm{mg}, 9.8 \square \mathrm{mol})$ and the resulting mixture was left to stir at $\mathrm{rt}$. After $17 \mathrm{~h}$ an additional $10 \mathrm{~mol} \% \mathrm{AgNO}_{3}$ was added and the reaction mixture was maintained at $\mathrm{rt}$ for $19 \mathrm{~h}$. The solution was concentrated and the crude oil purified by flash column chromatography (50:1 to 10:1 hexanes:EtOAc eluent) to afford unreacted $7\left(27 \mathrm{mg}, 69 \%\right.$ yield, $\mathrm{R}_{f}=0.39$ in $9: 1$ hexanes:EtOAc, $>20: 1 \mathrm{dr})$ and the desired pyran $16\left(10 \mathrm{mg}, 26 \%\right.$ yield, $\mathrm{R}_{f}=0.68$ in 9:1 hexanes:EtOAc, $\left.>20: 1 \mathrm{dr}\right)$ as a colorless oil: ${ }^{1} \mathrm{H}$ NMR $\left(400 \mathrm{MHz}, \mathrm{C}_{6} \mathrm{D}_{6}\right) \square 7.20-7.15(\mathrm{~m}, 2 \mathrm{H}), 7.10-7.07(\mathrm{~m}, 3 \mathrm{H}), 5.18(\mathrm{~d}, 1 \mathrm{H}, \mathrm{J}=0.8 \mathrm{~Hz}), 4.38$ $(\mathrm{d}, 1 \mathrm{H}, J=10.8 \mathrm{~Hz}), 4.08$ (br q, $1 \mathrm{H}, J=6.8 \mathrm{~Hz}), 3.92(\mathrm{t}, 1 \mathrm{H}, J=9.2 \mathrm{~Hz}), 3.80$ (dd, 1H, $J=9.6,3.6 \mathrm{~Hz}), 3.49$ (dd, $1 \mathrm{H}, J=11.2,2.0 \mathrm{~Hz}$ ), 2.50-2.44 (m, 2H), 2.04-2.02 (br m, 1H), 1.96 (br t, 2H, J=7.6 Hz), 1.72-1.60 (m, 2H), 1.19 $(\mathrm{t}, 3 \mathrm{H}, J=6.4 \mathrm{~Hz}), 1.14-1.04(\mathrm{~m}, 21 \mathrm{H}) ;{ }^{13} \mathrm{C} \mathrm{NMR}\left(100 \mathrm{MHz}, \mathrm{C}_{6} \mathrm{D}_{6}\right) \square 142.2,135.6,128.4,128.3,127.6,125.8,70.5$, 64.8, 63.7, 41.5, 35.6, 34.7, 29.5, 21.7, 18.0, 12.0; HRMS $\left(\mathrm{EI}^{+}\right)$calc'd for $\left[\mathrm{C}_{25} \mathrm{H}_{42} \mathrm{O}_{2} \mathrm{Si}_{-}-\mathrm{C}_{3} \mathrm{H}_{6}\right]^{+}: \mathrm{m} / z$ 360.2485, found 360.2433 .

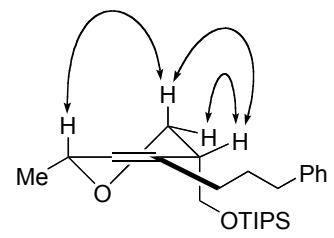

Selected nOe cross peaks for pyran $\mathbf{1 6}$ 


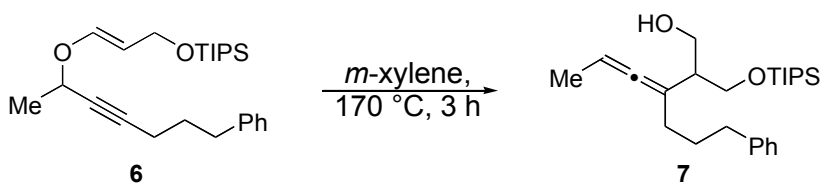

A solution of $6(50 \mathrm{mg}, 0.12 \mathrm{mmol})$ in m-xylene $(0.36 \mathrm{~mL})$ was heated to $170{ }^{\circ} \mathrm{C}$ and maintained for $3 \mathrm{~h}$. The reaction mixture was cooled to $\mathrm{rt}$, diluted with $\mathrm{MeOH}(0.72 \mathrm{~mL})$ and $\mathrm{NaBH}_{4}(5.4 \mathrm{mg}, 0.12 \mathrm{mmol})$ was added. After $1 \mathrm{~h}$ at $\mathrm{rt}$ the reaction mixture was filtered through silica gel washing with $\mathrm{Et}_{2} \mathrm{O}(5 \mathrm{~mL})$ and concentrated to afford homoallenic alcohol 7 (45 mg, 90\% yield, $\mathrm{R}_{f}=0.27$ in 9:1 hexanes:EtOAc, 1.5:1.0 mixture of diastereomers) as a pale yellow oil: ${ }^{1} \mathrm{H} \mathrm{NMR}\left(500 \mathrm{MHz}, \mathrm{CDCl}_{3}\right)$ The following were observed for the mixture of diastereomers $\square 7.29$ $7.25(\mathrm{~m}, 2 \mathrm{H}), 7.19-7.16(\mathrm{~m}, 3 \mathrm{H}), 5.16-5.13(\mathrm{~m}, 1 \mathrm{H}), 3.91-3.88(\mathrm{~m}, 1 \mathrm{H}), 3.79-3.69(\mathrm{~m}, 3 \mathrm{H}), 2.62(\mathrm{t}, 2 \mathrm{H}, J=7.5 \mathrm{~Hz})$, 2.31-2.26 (m, 1H), 2.02-1.99 (m, 2H), 1.73 (quintet, $2 \mathrm{H}, J=7.5 \mathrm{~Hz}), 1.64(\mathrm{t}, 3 \mathrm{H}, J=7.0 \mathrm{~Hz}), 1.12-1.02(\mathrm{~m}, 21 \mathrm{H})$; ${ }^{13} \mathrm{C} \mathrm{NMR}\left(100 \mathrm{MHz}, \mathrm{CDCl}_{3}\right)$ The following were observed for the major diastereomer $\square$ 100.9, 66.3, 46.0, 31.8, 15.1; The following were observed for the minor diastereomer $\square$ 100.9, 66.0, 46.1, 31.7, 15.0; The following were observed for the mixture of diastereomers $\square 201.8,142.4,128.5,128.3,125.8,88.4,67.2,35.4,29.4,18.0,11.8$.

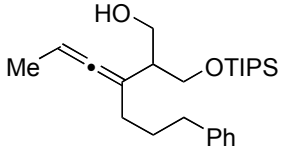

7

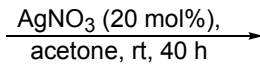

acetone, rt, $40 \mathrm{~h}$

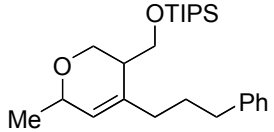

16

The Ag(I)-catalyzed cyclization was carried out using the modification of the published procedure described above. ${ }^{7}$ The crude oil was purified by flash column chromatography (100:1 hexanes:EtOAc eluent) to afford the desired pyran 16 (5.2 mg, 12\% yield, $\mathrm{R}_{f}=0.68$ in 9:1 hexanes:EtOAc, 1.4:1.0 mixture of diastereomers) as a colorless oil: ${ }^{1} \mathrm{H}$ NMR $\left(500 \mathrm{MHz}, \mathrm{C}_{6} \mathrm{D}_{6}\right)$ The following were observed for the major diastereomer $\square 5.23(\mathrm{~s}, 1 \mathrm{H}), 4.19$ (br q, $1 \mathrm{H}, J$ $=7.0 \mathrm{~Hz}), 3.91-3.89(\mathrm{~m}, 2 \mathrm{H}), 3.70-3.66(\mathrm{~m}, 2 \mathrm{H}), 2.22(\mathrm{~m}, 1 \mathrm{H})$; The following were observed for the minor diastereomer $\square 5.15(\mathrm{~s}, 1 \mathrm{H}), 4.35(\mathrm{~d}, 1 \mathrm{H}, J=11.0 \mathrm{~Hz}), 4.05(\mathrm{br} \mathrm{q}, 1 \mathrm{H}, J=7.0 \mathrm{~Hz}), 3.90(\mathrm{~m}, 1 \mathrm{H}), 3.76(\mathrm{dd}, 1 \mathrm{H}, J=$ 9.5, $3.5 \mathrm{~Hz}), 3.46(\mathrm{dd}, 1 \mathrm{H}, J=11.0,1.5 \mathrm{~Hz})$; The following were observed for the mixture of diastereomers $\square 7.16-$ $7.12(\mathrm{~m}, 2 \mathrm{H}), 7.07-7.04(\mathrm{~m}, 3 \mathrm{H}), 2.51-2.39(\mathrm{~m}, 2 \mathrm{H}), 1.99-1.90(\mathrm{~m}, 3 \mathrm{H}), 1.69-1.57$ (m, 2H), $1.15(\mathrm{t}, 3 \mathrm{H}, J=7.0 \mathrm{~Hz})$, $1.10-0.99(\mathrm{~m}, 21 \mathrm{H})$.

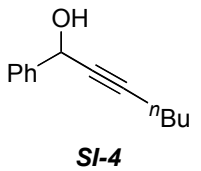

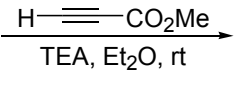

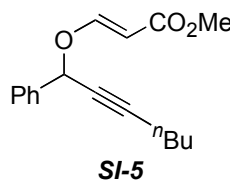

SI-5

The preparation of SI-5 was carried out according to the method of Ireland and Wipf. ${ }^{8}$ The crude red oil was purified by flash column chromatography (12:1 hexanes:EtOAc eluent) to afford the desired vinylogous carbonate SI-5 (1.36 g, 94\% yield, $\mathrm{R}_{f}=0.65$ in 3:1 hexanes:EtOAc) as a pale yellow liquid: ${ }^{1} \mathrm{H} \mathrm{NMR}\left(400 \mathrm{MHz}^{\mathrm{CDCl}} \mathrm{CD}_{3}\right.$ $7.70(\mathrm{~d}, 1 \mathrm{H}, J=12.4 \mathrm{~Hz}), 7.50(\mathrm{~d}, 2 \mathrm{H}, J=6.4 \mathrm{~Hz}), 7.41-7.34(\mathrm{~m}, 3 \mathrm{H}), 5.63(\mathrm{~s}, 1 \mathrm{H}), 5.44(\mathrm{~d}, 1 \mathrm{H}, J=12.8 \mathrm{~Hz}), 3.69$ $(\mathrm{s}, 3 \mathrm{H}), 2.30(\mathrm{td}, 2 \mathrm{H}, J=6.8,1.6 \mathrm{~Hz}), 1.53$ (quintet, $2 \mathrm{H}, J=6.8 \mathrm{~Hz}), 1.41($ sextet, $2 \mathrm{H}, J=7.2 \mathrm{~Hz}), 0.91(\mathrm{t}, 3 \mathrm{H}, J=$ $7.6 \mathrm{~Hz}) ;{ }^{13} \mathrm{C} \mathrm{NMR}\left(100 \mathrm{MHz}, \mathrm{CDCl}_{3}\right) \square 168.1,160.2,137.0,129.1,128.7,127.5,99.1,91.5,75.6,73.8,51.1,30.4$, 22.0, 18.5, 13.6; HRMS $\left(\mathrm{EI}^{+}\right)$calc'd for $\left[\mathrm{C}_{17} \mathrm{H}_{20} \mathrm{O}_{3}\right]^{+}: \mathrm{m} / \mathrm{z} 272.1412$, found 272.1408.
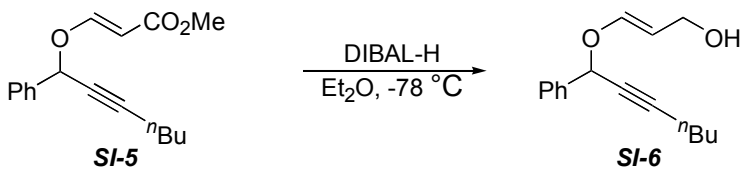

The reduction of $\boldsymbol{S I - 5}$ was carried out according to the method of Ireland and Wipf. ${ }^{8}$ The crude oil was purified by flash column chromatography (2\% TEA in 4:1 hexanes:EtOAc eluent) to afford the desired alcohol SI-6 (0.618 g, $69 \%$ yield, $\mathrm{R}_{f}=0.26$ in $3: 1$ hexanes:EtOAc) as a pale yellow oil: ${ }^{1} \mathrm{H}$ NMR $\left(400 \mathrm{MHz}, \mathrm{CDCl}_{3}\right) \square 7.50(\mathrm{~d}, 2 \mathrm{H}, J=7.2$ $\mathrm{Hz}), 7.39-7.31(\mathrm{~m}, 3 \mathrm{H}), 6.56(\mathrm{~d}, 1 \mathrm{H}, J=12.4 \mathrm{~Hz}), 5.48(\mathrm{~s}, 1 \mathrm{H}), 5.30(\mathrm{dt}, 1 \mathrm{H}, J=12.4,7.6 \mathrm{~Hz}), 4.04(\mathrm{~d}, 2 \mathrm{H}, J=7.2$ $\mathrm{Hz}$ ), 2.28 (td, $2 \mathrm{H}, J=6.8,1.6 \mathrm{~Hz}), 1.53$ (quintet, $2 \mathrm{H}, J=6.8 \mathrm{~Hz}), 1.41$ (sextet, $2 \mathrm{H}, J=7.2 \mathrm{~Hz}), 0.91(\mathrm{t}, 3 \mathrm{H}, J=7.2$ $\mathrm{Hz}), 1.25$ (br s, $1 \mathrm{H}) ;{ }^{13} \mathrm{C}$ NMR (100 MHz, $\left.\mathrm{CDCl}_{3}\right) \square 147.5,138.0,128.6,128.5,127.3,106.3,89.9,76.7,71.9,60.6$, 30.5, 21.9, 18.5, 13.5; HRMS $\left(\mathrm{EI}^{+}\right)$calc'd for $\left[\mathrm{C}_{16} \mathrm{H}_{20} \mathrm{O}_{2}-\mathrm{CH}_{3} \mathrm{O}\right]^{+}: \mathrm{m} / z$ 213.1279, found 213.1280. 

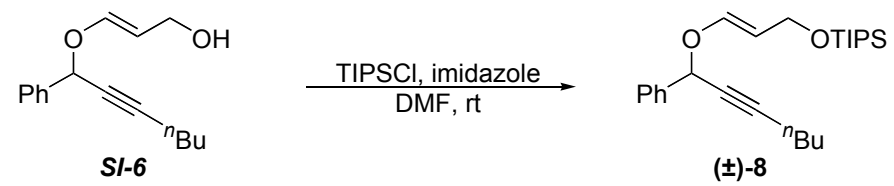

To a solution of $\boldsymbol{S I - 6}(0.150 \mathrm{~g}, 0.614 \mathrm{mmol})$ in DMF $(1.5 \mathrm{~mL})$ was added imidazole $(96 \mathrm{mg}, 1.4 \mathrm{mmol})$ followed by TIPSCl $(0.16 \mathrm{~mL}, 0.74 \mathrm{mmol})$ and the resulting solution was left to stir at rt. TLC of the reaction mixture after $18 \mathrm{~h}$ showed consumption of starting alcohol and the reaction mixture was diluted with $\mathrm{H}_{2} \mathrm{O}(5 \mathrm{~mL})$. The solution was extracted with $\mathrm{Et}_{2} \mathrm{O}(3 \times 5 \mathrm{~mL})$, and the combined organic layers washed with brine $(3 \times 10 \mathrm{~mL})$, dried over $\mathrm{MgSO}_{4}$, filtered, and concentrated. The crude reside was purified by flash column chromatography ( $2 \%$ TEA in hexanes eluent) to afford the desired silyl ether $( \pm)-8\left(204 \mathrm{mg}, 83 \%\right.$ yield, $\mathrm{R}_{f}=0.89$ in 3:1 hexanes:EtOAc) as a colorless oil: ${ }^{1} \mathrm{H}$ NMR $\left(400 \mathrm{MHz}, \mathrm{CDCl}_{3}\right) \square 7.50(\mathrm{~d}, 2 \mathrm{H}, J=6.8 \mathrm{~Hz}), 7.38-7.31(\mathrm{~m}, 3 \mathrm{H}), 6.53(\mathrm{~d}, 1 \mathrm{H}, J=12.4 \mathrm{~Hz}), 5.46(\mathrm{~s}, 1 \mathrm{H})$, $5.22(\mathrm{dt}, 1 \mathrm{H}, J=12.4,6.4 \mathrm{~Hz}), 4.18(\mathrm{~d}, 2 \mathrm{H}, J=6.4 \mathrm{~Hz}), 2.27(\mathrm{td}, 2 \mathrm{H}, J=7.2,2.0 \mathrm{~Hz}), 1.55-1.48(\mathrm{~m}, 2 \mathrm{H}), 1.44-1.38$ $(\mathrm{m}, 2 \mathrm{H}), 1.08-1.02(\mathrm{~m}, 21 \mathrm{H}), 0.90(\mathrm{t}, 3 \mathrm{H}, J=7.6 \mathrm{~Hz}) ;{ }^{13} \mathrm{C}$ NMR $\left(100 \mathrm{MHz}, \mathrm{CDCl}_{3}\right) \square 145.6,138.3,128.5,127.4$, 107.0, 89.6, 71.8, 61.1, 30.6, 22.0, 18.6, 18.0, 13.6, 12.1 .<smiles>OCC(CO[Na])C(Br)=C=Cc1ccccc1</smiles>

$( \pm)-9$

The Claisen rearrangement was carried out following the general procedure described above. The crude mixture was purified by flash column chromatography (12:1 hexanes:EtOAc eluent) to afford the desired homoallenic alcohol $\left(167 \mathrm{mg}, 89 \%\right.$ yield, $\mathrm{R}_{f}=0.36$ in 9:1 hexanes:EtOAc) as a colorless oil: ${ }^{1} \mathrm{H}$ NMR $\left(400 \mathrm{MHz}, \mathrm{CDCl}_{3}\right) \square$ 7.29-7.22 (m, 4H), 7.19-7.15 (m, 1H), $6.18(\mathrm{q}, 1 \mathrm{H}, J=1.6 \mathrm{~Hz}), 4.02(\mathrm{dd}, 1 \mathrm{H}, J=10.0,4.4 \mathrm{~Hz}), 3.83-3.76(\mathrm{~m}, 3 \mathrm{H})$, 2.95 (br s, $1 \mathrm{H}), 2.49-2.45(\mathrm{~m}, 1 \mathrm{H}), 2.15-2.10(\mathrm{~m}, 2 \mathrm{H}), 1.49-1.41(\mathrm{~m}, 2 \mathrm{H}), 1.38-1.31(\mathrm{~m}, 2 \mathrm{H}), 1.10-1.0(\mathrm{~m}, 21 \mathrm{H})$, $0.87(\mathrm{t}, 3 \mathrm{H}, J=7.2 \mathrm{~Hz}) ;{ }^{13} \mathrm{C}$ NMR $\left(100 \mathrm{MHz}, \mathrm{CDCl}_{3}\right) \square 202.1,135.2,128.6,126.8,126.4,106.5,97.0,67.1,66.0$, 46.8, 32.3, 29.8, 22.5, 17.9, 12.9, 11.8; HRMS $\left(\mathrm{EI}^{+}\right)$calc'd for $\left[\mathrm{C}_{25} \mathrm{H}_{42} \mathrm{O}_{2} \mathrm{Si}\right]^{+}: m / z$ 402.2954, found 402.2955.<smiles>OCC(COc1ccccc1)C(Br)=CC=Cc1ccccc1</smiles>

$( \pm)-9$

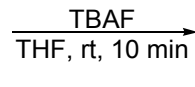

To a solution of $( \pm)-9(30 \mathrm{mg}, 74 \square \mathrm{mol})$ in THF $(0.2 \mathrm{~mL})$ was added TBAF $(0.1 \mathrm{~mL}, 0.10 \mathrm{mmol}, 1.0 \mathrm{M}$ in THF), and the resulting solution was maintained at $\mathrm{rt}$ for $10 \mathrm{~min}$. The crude mixture was purified by flash column chromatography (2:1 hexanes:EtOAc eluent) to afford the desired diol $\left(7 \mathrm{mg}, 50 \%\right.$ yield, $\mathrm{R}_{f}=$ in $3: 1$ hexanes:EtOAc) as a white solid: ${ }^{1} \mathrm{H}$ NMR $\left(400 \mathrm{MHz}, \mathrm{CDCl}_{3}\right) \square 7.31-7.25(\mathrm{~m}, 4 \mathrm{H}), 7.20-7.17(\mathrm{~m}, 1 \mathrm{H}), 6.23$ (br q, $1 \mathrm{H}, J=1.6 \mathrm{~Hz}), 3.94-3.87(\mathrm{td}, 2 \mathrm{H}, J=11.2,4.4 \mathrm{~Hz}), 3.83-3.78(\mathrm{~m}, 2 \mathrm{H}), 2.44-2.42(\mathrm{~m}, 1 \mathrm{H}), 2.17-2.11(\mathrm{~m}, 4 \mathrm{H}), 1.50-$ $1.40(\mathrm{~m}, 2 \mathrm{H}), 1.35$ (sextet, $2 \mathrm{H}, J=7.2 \mathrm{~Hz}), 0.87(\mathrm{t}, 3 \mathrm{H}, J=7.2 \mathrm{~Hz}) ;{ }^{13} \mathrm{C}$ NMR $\left(100 \mathrm{MHz}, \mathrm{CDCl}_{3}\right) \square 202.1,134.9$, $128.8,127.0,126.4,106.7,97.1,64.9,46.6,32.0,29.8,22.5,13.9$.<smiles>OCC(CO[18OH])C(Br)=Cc1ccccc1</smiles>

9

The Claisen rearrangement was carried out following the general procedure described above. The crude mixture was purified by flash column chromatography ( $9: 1$ hexanes:EtOAc eluent) to afford the desired homoallenic alcohol (122 mg, 81\% yield, $\mathrm{R}_{f}=0.26$ in 9:1 hexanes:EtOAc, $>20: 1 \mathrm{dr}$ ) as a colorless oil: Spectral data were consistent with those reported for $( \pm)-9$.

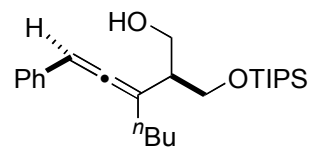

9

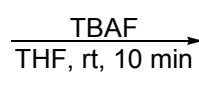

(a)

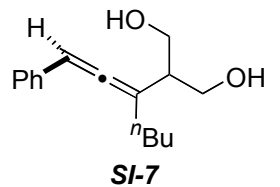

SI-7

Desilylation was carried out using the procedure described above for the preparation ( \pm )-SI-7. The crude mixture was purified by flash column chromatography (2:1 hexanes:EtOAc eluent) to afford the desired diol $\mathbf{S I - 7}$ (66 $\mathrm{mg}$, 
90\% yield, $\mathrm{R}_{f}=0.05$ in 3:1 hexanes:EtOAc) as a white solid: Spectral data were consistent with those reported for ( \pm -SI-7; Enantiomeric purity was determined by chiral HPLC analysis, Chiralcel OD column, 92:8 hexanes:isopropanol, $1 \mathrm{~mL} / \mathrm{min}$; $\mathrm{t}_{\mathrm{R}} 14.99 \mathrm{~min}$ (minor), $17.43 \mathrm{~min}$ (major): 94\% ee.

\section{Allyl / Propargyl Competition Experiment:}<smiles>C=CC=C=C(P)CCO</smiles>

13

The Claisen rearrangement was carried out following the general procedure described above. The crude mixture was purified by flash column chromatography ( $9: 1$ hexanes:EtOAc eluent) to afford the desired homoallenic alcohol ( $81 \mathrm{mg}, 80 \%$ yield, $\mathrm{R}_{\mathrm{f}}=0.24$ in $3: 1$ hexanes:EtOAc) as a yellow oil: ${ }^{1} \mathrm{H}$ NMR $\left(400 \mathrm{MHz}, \mathrm{CDCl}_{3}\right) \square 7.40(\mathrm{~d}, 2 \mathrm{H}, J=$ $8.0 \mathrm{~Hz}), 7.33(\mathrm{t}, 2 \mathrm{H}, J=7.2 \mathrm{~Hz}), 7.23(\mathrm{t}, 1 \mathrm{H}, J=7.2 \mathrm{~Hz}), 6.30-6.20(\mathrm{~m}, 2 \mathrm{H}), 5.35-5.28(\mathrm{~m}, 1 \mathrm{H}), 5.09(\mathrm{~d}, 1 \mathrm{H}, J=8.8$ $\mathrm{Hz}), 3.85(\mathrm{t}, 2 \mathrm{H}, J=6.4 \mathrm{~Hz}), 2.79-2.70(\mathrm{~m}, 2 \mathrm{H}), 2.02(\mathrm{br} \mathrm{s}, 1 \mathrm{H}) ;{ }^{13} \mathrm{C} \mathrm{NMR}\left(100 \mathrm{MHz}, \mathrm{CDCl}_{3}\right) \square 207.7,135.7,132.4$, 128.3, 127.3, 126.2, 117.0, 104.2, 98.4, 61.1, 33.3; HRMS $\left(\mathrm{EI}^{+}\right)$calc'd for $\left[\mathrm{C}_{13} \mathrm{H}_{14} \mathrm{O}\right]^{+}: \mathrm{m} / z$ 186.1045, found 186.1045 .

\section{Examination of $\mathrm{Au}(\mathrm{I})-$ Species Generated Under the Reaction Conditions:}

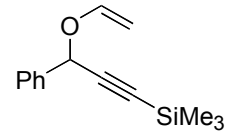

$( \pm)-4 n$

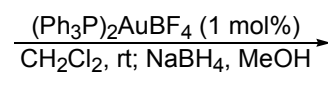

$\mathrm{CH}_{2} \mathrm{Cl}_{2}$, rt; $\mathrm{NaBH}_{4}, \mathrm{MeOH}$

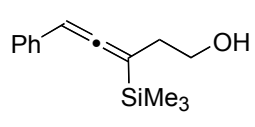

$( \pm)-5 n$

To a solution of $( \pm)-4 \mathbf{n}(0.100 \mathrm{~g}, 0.434 \mathrm{mmol})$ in $\mathrm{CH}_{2} \mathrm{Cl}_{2}(1.24 \mathrm{~mL})$ was added $\left(\mathrm{Ph}_{3} \mathrm{P}\right)_{2} \mathrm{AuBF}_{4}(3.5 \mathrm{mg}, 4.3 \square \mathrm{mol})$ and the resulting solution was left to stir at rt and monitored by TLC. After $18 \mathrm{~h}$ the mixture was concentrated, redissolved in $\mathrm{MeOH}(1.24 \mathrm{~mL})$, and $\mathrm{NaBH}_{4}(15 \mathrm{mg}, 0.39 \mathrm{mmol})$ was added. TLC analysis of the reaction mixture after $1 \mathrm{~h}$ indicated consumption of the intermediate aldehyde and the solution was concentrated. The crude red oil was purified by flash column chromatography (9:1 hexanes:EtOAc eluent) to afford the desired homoallenic alcohol (67 mg, 66\% yield, $\mathrm{R}_{f}=0.36$ in 3:1 hexanes:EtOAc) as a colorless oil: Spectral data were consistent with those reported for $\mathbf{( S ) - 5 n . ~}$

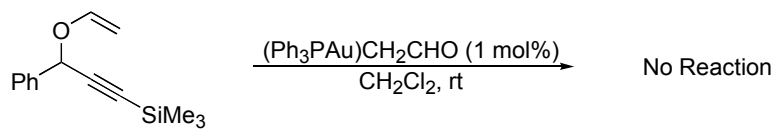

$( \pm)-4 n$

To a solution of $( \pm)-4 \mathbf{n}(20 \mathrm{mg}, 87$ mol $)$ in $\mathrm{CH}_{2} \mathrm{Cl}_{2}(0.25 \mathrm{~mL})$ was added $\left(\mathrm{Ph}_{3} \mathrm{PAu}\right) \mathrm{CH}_{2} \mathrm{CHO}(1.3 \mathrm{mg}, 2.6$ mol $)$ and the reaction mixture was left to stir at $\mathrm{rt}$ and monitored by TLC. After $20 \mathrm{~h}$ the mixture was concentrated and a crude ${ }^{1} \mathrm{H}$ spectrum indicated only the starting vinyl ether $( \pm)-\mathbf{4 n}$ present in solution: ${ }^{1} \mathrm{H}$ NMR $\left(400 \mathrm{MHz}, \mathrm{CDCl}_{3}\right) \square$ $7.53(\mathrm{~d}, 2 \mathrm{H}, J=6.8 \mathrm{~Hz}), 7.41-7.33(\mathrm{~m}, 2 \mathrm{H}), 6.50(\mathrm{dd}, 1 \mathrm{H}, J=14.0,6.4 \mathrm{~Hz}), 5.50(\mathrm{~s}, 1 \mathrm{H}), 4.51(\mathrm{dd}, 1 \mathrm{H}, J=14.0,1.6$ $\mathrm{Hz}), 4.18(\mathrm{dd}, 1 \mathrm{H}, J=6.8,2.0 \mathrm{~Hz}), 0.19(\mathrm{~s}, 9 \mathrm{H}) ;{ }^{13} \mathrm{C}$ NMR $\left(100 \mathrm{MHz}, \mathrm{CDCl}_{3}\right) \mathrm{1}$ 149.3, 137.4, 128.6, 128.5, 127.4, 101.6, 93.7, 90.6, 71.1, -0.2; HRMS $\left(\mathrm{EI}^{+}\right)$calc'd for $\left[\mathrm{C}_{14} \mathrm{H}_{18} \mathrm{OSi}\right]^{+}: \mathrm{m} / \mathrm{z} 230.1127$, found 230.1127; Anal calc'd for $\mathrm{C}_{14} \mathrm{H}_{18} \mathrm{OSi}$ : C, 72.99; H, 7.88; found: C, 72.86; H, 7.83.

\section{References:}

(1) Bruce, M.I.; Nicholson, B.K.; Bin Shawkataly, O. Inorg. Syn. 1989, 26, 324.

(2) (a) Yang, Y.; Ramamoorthy, V.; Sharp, P.R. Inorg. Chem. 1993, 32, 1946. (b) Nesmeyanov, A.N.; Perevalova, E.G.; Struchkov, Yu. T.; Antipin, M. Yu.; Grandberg, K.I.; Dyadchenko, V.P. J. Organomet. Chem. 1980, $201,343$.

(3) Westland, A.D. Can. J. Chem. 1969, 47, 4135.

(4) Nesmeyanov, A.N.; Perevalova, E.G.; Dyadchenko, V.P.; Grandberg, K.I. Izvestiya Akademii Nauk SSSR, Seriya Khimicheskaya 1974, 12, 2872.

(5) Midland, M.M.; Tramontano, A.; Kazubski, A.; Graham, R.S.; Tsai, D.J.S.; Cardin, D.B. Tetrahedron 1984, 40, 1371 .

(6) Nonoshita, K.; Banno, H.; Maruoka, K.; Yamamoto, H. J. Am. Chem. Soc. 1990, 112, 316.

(7) Wan, Z.; Nelson, S.G. J. Am. Chem. Soc. 2000, 122, 10470.

(8) Ireland, R.E.; Wipf, P.; Xiang, J.-N. J. Org. Chem. 1991, 56, 3572. 